\title{
HAANTJES ALGEBRAS AND DIAGONALIZATION
}

\author{
PIERGIULIO TEMPESTA AND GIORGIO TONDO
}

\begin{abstract}
We introduce the notion of Haantjes algebra: It consists of an assignment of a family of operator fields on a differentiable manifold, each of them with vanishing Haantjes torsion. They are also required to satisfy suitable compatibility conditions. Haantjes algebras naturally generalize several known interesting geometric structures, arising in Riemannian geometry and in the theory of integrable systems. At the same time, as we will show, they play a crucial role in the theory of diagonalization of operators on differentiable manifolds. Assuming that the operators of a Haantjes algebra are semisimple and commute, we shall prove that there exists a set of local coordinates where all operators can be diagonalized simultaneously. Moreover, in the general, non-semisimple case, they acquire simultaneously, in a suitable local chart, a block-diagonal form.
\end{abstract}

\section{Contents}

1. Introduction

2. Nijenhuis and Haantjes operators

2.1. Algebraic preliminaries

2.2. A new family of generalized Nijenhuis torsions

2.3. General properties of Haantjes operators

2.4. An application to Classical Mechanics: the inertia tensor

3. The geometry of Haantjes operators

3.1. Integrability

3.2. A new proof of the Haantjes theorem

4. Characteristic Haantjes coordinates and block diagonalization 14

5. Haantjes algebras 17

5.1. Main Definition 17

5.2. Cyclic Haantjes algebras 20

6. Haantjes algebra for the Coulomb-Kepler potential 23

7. Cyclic Haantjes operators: Example 24

7.1. Construction of a Haantjes chart 24

8. A comparison with other algebraic structures: Haantjes manifolds and Killing-Stäckel algebras

Acknowledgments

References

Date: November 05, 2020.

2010 Mathematics Subject Classification. MSC: 53A45, 58C40.

Key words and phrases. Haantjes tensors, Haantjes manifolds, generalized Nijenhuis torsions. 


\section{INTRODUCTION}

The purpose of this paper is to introduce a new geometric-algebraic structure, based on the notion of Haantjes torsion, that we shall call Haantjes algebra. The Haantjes torsion was introduced in 1955 by J. Haantjes in [15], as a natural generalization of the torsion defined by Nijenhuis in [27. The theory of tensor fields with vanishing Nijenhuis torsion has been intensively investigated in the last forty years, mainly due to its relevance in the theory of almost complex structures [26] and its applications to the theory of integrable systems and separation of variables, where they are usually called recursion operators [13, 9, 24, 16, 10, 8. However, quite surprisingly, the relevance of Haantjes's differential-geometric work has not been recognized for a long time, except for some notable applications to Hamiltonian systems of hydrodynamic type [25, 6, 7, 11, 14. For a nice review of classical and more recent results regarding the theory of Nijenhuis and Haantjes tensors, see Ref. [18].

Our work is inspired, one the one hand, by the construction of Haantjes manifolds proposed in [19, 20, 23]; on the other hand, by the concept of $\omega \mathcal{H}$ (or symplecticHaantjes) manifolds that we have recently introduced in [29] in connection with the theory of classical integrable systems. In this context, $\omega \mathcal{H}$ structures provide us with a natural theoretical framework for dealing with the integrability and separability properties of Hamiltonian systems, which parallels and completes the approach offered by the Nijenhuis geometry.

In this article, we will extend the previous ideas by proposing the general and abstract notion of Haantjes algebras. They consist essentially of a differentiable manifold $M$ endowed with a family of endomorphisms of the tangent bundle with vanishing Haantjes torsion, which are compatible with each other. In this more general framework, the existence of an underlying symplectic structure is no longer required. In this work, we shall focus on operators with real eigenvalues only.

A Haantjes algebra is a flexible tool that can be specialized to treat many different interesting constructions in a natural and unified language. For example, Magri's Haantjes manifolds are a specially relevant instance of Haantjes algebras. Another important class of Haantjes algebras is represented by the Killing-Stäckel algebras introduced in [3] on a Riemannian manifold, with the aim to characterize separation of variables in classical Hamiltonian systems.

However, apart from the intrinsic interest of structures combining the Haantjes geometry with symplectic or Riemannian geometry, our main motivation is the abstract problem of the diagonalization of operators on a differentiable manifold. Indeed, we shall prove that the algebras of Haantjes operators introduced in Section 4 can be diagonalized simultaneously in a suitable local coordinate system, called a Haantjes chart. Note that no hermiticity assumption is needed: we only require that the Haantjes operators are a set of pointwise diagonalizable commuting operators. Since the vanishing of the Haantjes torsion of an operator is a fourth-degree requirement in its components (see formulae (4) and (5)), a very large class of tensor fields satisfies it. In Theorem 43 we prove the following

Main Result. Given a semisimple Abelian Haantjes algebra $\mathscr{H}$ on a manifold $M$, that is an algebra of semisimple commuting operator fields with vanishing Haantjes torsion, there exist sets of local coordinates on $M$ in which all the operators can be simultaneously diagonalized. 
Conversely, let $\mathcal{K}$ be a family of commuting semisimple operator fields. If they share a set of local coordinates in which they take simultaneously a diagonal form, then they generate a semisimple Abelian Haantjes algebra.

The previous result can also be extended to the very general (but much less explored) case of non-semisimple Haantjes operators. Indeed, for this class we shall prove that there exists a local coordinate system where all the operators of a set of commuting Haantjes operators acquire simultaneously a block-diagonal form.

In this article a new, infinite "tower" of generalized Nijenhuis torsions of level $n$ for all $n \in \mathbb{N}$ is also defined. The geometrical meaning of our notion, which naturally generalizes both the classical Nijenhuis and Haantjes torsions, has been discussed in detail in Ref. 30.

The paper is organized as follows. After a discussion, in Section 2, of the main algebraic structures relevant for this work, including the generalized torsions, we present in Section 3 a brief introduction to the geometry of Nijenhuis and Haantjes tensors. Also, some new results concerning non-semisimple Haantjes operators are proposed. They allow us to derive the classical Haantjes theorem 15 from a new perspective. In Section 4, we discuss the block diagonalization of commuting Haantjes operators. In Section 5, the formal construction of a Haantjes algebra is introduced and the cyclic case is discussed. The results concerning the simultaneous diagonalization of semisimple commuting Haantjes operators are also proved. In Section 6, the Haantjes structure for the classical Coulomb-Kepler system is discussed. A related example, illustrating the application of the main theorems and the theory of cyclic generators of semisimple Haantjes algebras, is presented in Section 7 .

A comparative discussion of other geometric structures related with Haantjes algebras is proposed in the final Section 8.

\section{NiJenhuis And HaAntJes operators}

The natural frames of vector fields associated with local coordinates on a differentiable manifold, being obviously integrable, can be characterized in a tensorial manner as eigen-distributions of a suitable class of $(1,1)$ tensor fields, i.e. the ones with vanishing Nijenhuis or Haantjes torsion. In this section, we review some basic algebraic results concerning the theory of such tensors. For a more complete treatment, see the original papers [15, 27] and the related ones [28, 12].

2.1. Algebraic preliminaries. Let $M$ be a real differentiable manifold and $\boldsymbol{L}$ : $T M \rightarrow T M$ a smooth $(1,1)$ tensor field, i.e., a field of linear operators on the tangent space at each point of $M$. In the following, all tensors will be assumed to be smooth.

Definition 1. The Nijenhuis torsion of $\boldsymbol{L}$ is defined by the vector-valued 2-form

$$
\mathcal{T}_{\boldsymbol{L}}(X, Y):=\boldsymbol{L}^{2}[X, Y]+[\boldsymbol{L} X, \boldsymbol{L} Y]-\boldsymbol{L}([X, \boldsymbol{L} Y]+[\boldsymbol{L} X, Y]),
$$

where $X, Y \in T M$ and [, ] denotes the commutator of two vector fields.

In local coordinates $\boldsymbol{x}=\left(x^{1}, \ldots, x^{n}\right)$, the Nijenhuis torsion can be written as the skew-symmetric $(1,2)$ tensor field

$$
\left(\mathcal{T}_{\boldsymbol{L}}\right)_{j k}^{i}=\sum_{\alpha=1}^{n}\left(\frac{\partial \boldsymbol{L}_{k}^{i}}{\partial x^{\alpha}} \boldsymbol{L}_{j}^{\alpha}-\frac{\partial \boldsymbol{L}_{j}^{i}}{\partial x^{\alpha}} \boldsymbol{L}_{k}^{\alpha}+\left(\frac{\partial \boldsymbol{L}_{j}^{\alpha}}{\partial x^{k}}-\frac{\partial \boldsymbol{L}_{k}^{\alpha}}{\partial x^{j}}\right) \boldsymbol{L}_{\alpha}^{i}\right)
$$


having $n^{2}(n-1) / 2$ independent components.

Definition 2. The Haantjes torsion associated with $\boldsymbol{L}$ is the vector-valued 2-form defined by

$$
\mathcal{H}_{\boldsymbol{L}}(X, Y):=\boldsymbol{L}^{2} \mathcal{T}_{\boldsymbol{L}}(X, Y)+\mathcal{T}_{\boldsymbol{L}}(\boldsymbol{L} X, \boldsymbol{L} Y)-\boldsymbol{L}\left(\mathcal{T}_{\boldsymbol{L}}(X, \boldsymbol{L} Y)+\mathcal{T}_{\boldsymbol{L}}(\boldsymbol{L} X, Y)\right)
$$

Explicitly, one can also write [18]

$$
\begin{aligned}
\mathcal{H}_{\boldsymbol{L}}(X, Y) & =\boldsymbol{L}^{4}[X, Y]-2 \boldsymbol{L}^{3}([X, \boldsymbol{L} Y]+[\boldsymbol{L} X, Y])+\boldsymbol{L}^{2}\left(\left[X, \boldsymbol{L}^{2} Y\right]+4[\boldsymbol{L} X, \boldsymbol{L} Y]+\left[\boldsymbol{L}^{2} X, Y\right]\right) \\
& -2 \boldsymbol{L}\left(\left[\boldsymbol{L} X, \boldsymbol{L}^{2} Y\right]+\left[\boldsymbol{L}^{2} X, \boldsymbol{L} Y\right]\right)+\left[\boldsymbol{L}^{2} X, \boldsymbol{L}^{2} Y\right]
\end{aligned}
$$

The skew-symmetry of the Nijenhuis torsion implies that the Haantjes torsion is also skew-symmetric. Its local expression is

$$
\left(\mathcal{H}_{\boldsymbol{L}}\right)_{j k}^{i}=\sum_{\alpha, \beta=1}^{n}\left(\boldsymbol{L}_{\alpha}^{i} \boldsymbol{L}_{\beta}^{\alpha}\left(\mathcal{T}_{\boldsymbol{L}}\right)_{j k}^{\beta}+\left(\mathcal{T}_{\boldsymbol{L}}\right)_{\alpha \beta}^{i} \boldsymbol{L}_{j}^{\alpha} \boldsymbol{L}_{k}^{\beta}-\boldsymbol{L}_{\alpha}^{i}\left(\left(\mathcal{T}_{\boldsymbol{L}}\right)_{\beta k}^{\alpha} \boldsymbol{L}_{j}^{\beta}+\left(\mathcal{T}_{\boldsymbol{L}}\right)_{j \beta}^{\alpha} \boldsymbol{L}_{k}^{\beta}\right)\right)
$$

or in explicit form

$$
\begin{aligned}
\left(\mathcal{H}_{\boldsymbol{L}}\right)_{j k}^{i} & =\sum_{\alpha=1}^{n}\left(-2\left(\boldsymbol{L}^{3}\right)_{\alpha}^{i} \partial_{[j} \boldsymbol{L}_{k]}^{\alpha}+\left(\boldsymbol{L}^{2}\right)_{\alpha}^{i}\left(\partial_{[j}\left(\boldsymbol{L}^{2}\right)_{k]}^{\alpha}+4 \sum_{\beta=1}^{n} \boldsymbol{L}_{[j}^{\beta} \partial_{|\beta|} \boldsymbol{L}_{k]}^{\alpha}\right)\right. \\
& \left.-2 \boldsymbol{L}_{\alpha}^{i}\left(\boldsymbol{L}_{[j}^{\beta} \partial_{|\beta|}\left(\boldsymbol{L}^{2}\right)_{k]}^{\alpha}+\left(\boldsymbol{L}^{2}\right)_{[j}^{\beta} \partial_{|\beta|}(\boldsymbol{L})_{k]}^{\alpha}\right)+\left(\boldsymbol{L}^{2}\right)_{[j}^{\alpha} \partial_{|\alpha|}\left(\boldsymbol{L}^{2}\right)_{k]}^{i}\right)
\end{aligned}
$$

Here for the sake of brevity we have used the notation $\partial_{j}:=\frac{\partial}{\partial x^{j}}$; the indices between square brackets are to be skew-symmetrized, except those in $|\cdot|$.

In order to prove some of the results of the following sections, we state preliminarily three lemmas.

Lemma 3. Let $\boldsymbol{L}: T M \rightarrow T M$ be an operator field. The following identities hold:

$$
\begin{aligned}
\tau_{\boldsymbol{L}}(X, Y) & =\boldsymbol{L}^{2}[X, Y], & & \forall X, Y \in \operatorname{ker}(\boldsymbol{L}) \\
\mathcal{H}_{\boldsymbol{L}}(X, Y) & =\boldsymbol{L}^{4}[X, Y], & & \forall X, Y \in \operatorname{ker}(\boldsymbol{L})
\end{aligned}
$$

Proof. These relations come directly from Eqs. (11) and (4), taking into account that only the first term is non-vanishing in their right hand sides, due to the fact that $X, Y \in \operatorname{ker}(\boldsymbol{L})$.

In the following result, we apply the classical Fitting lemma [17] to the module, over the ring $\mathcal{C}^{\infty}(M)$, of the operator fields on $T M$.

Lemma 4. Let $\boldsymbol{L}: T M \rightarrow T M$ be an operator field. For any $\boldsymbol{x} \in M$ there exists a minimum positive integer $\rho(\boldsymbol{x})$ such that

$$
\operatorname{ker} \boldsymbol{L}^{\rho(\boldsymbol{x})}(\boldsymbol{x})=\operatorname{ker} \boldsymbol{L}^{\rho(\boldsymbol{x})+1}(\boldsymbol{x}), \quad \operatorname{Im} \boldsymbol{L}^{\rho(\boldsymbol{x})}(\boldsymbol{x})=\operatorname{Im} \boldsymbol{L}^{\rho(\boldsymbol{x})+1}(\boldsymbol{x})
$$

and

$$
T_{\boldsymbol{x}} M=\operatorname{ker} \boldsymbol{L}^{\rho(\boldsymbol{x})}(\boldsymbol{x}) \oplus \operatorname{Im} \boldsymbol{L}^{\rho(\boldsymbol{x})}(\boldsymbol{x})
$$

The index $\rho(\boldsymbol{x})$ is said to be the Riesz index of $\boldsymbol{L}$ at $\boldsymbol{x}$. 
Lemma 5. Let $\boldsymbol{A}$ and $\boldsymbol{B}$ be two commuting operator fields with Riesz index $\rho(\boldsymbol{x})=$ 1 at any point $\boldsymbol{x}$ of $M$. Then, the identity

$$
\text { ker } \boldsymbol{A}+\operatorname{ker} \boldsymbol{B}=\operatorname{ker} \boldsymbol{A} \boldsymbol{B}
$$

holds.

Proof. The condition $\rho(\boldsymbol{x})=1$ is equivalent to the conditions ker $\boldsymbol{A}=\operatorname{ker} \boldsymbol{A}^{2}$ and ker $\boldsymbol{B}=\operatorname{ker} \boldsymbol{B}^{2}$. Besides, since the two operator fields $\boldsymbol{A}$ and $\boldsymbol{B}$ commute with each others, it is immediate to check that each vector field $Z:=X+Y$ with $X \in \operatorname{ker} \boldsymbol{A}$ and $Y \in \operatorname{ker} \boldsymbol{B}$ belongs to ker $\boldsymbol{A} \boldsymbol{B}$.

Conversely, let us suppose that $\operatorname{ker} \boldsymbol{A}=\operatorname{ker} \boldsymbol{A}^{2}$. Then, by means of Lemma 4 each $Z \in T M$ can be decomposed as $Z:=X+\boldsymbol{A} W$ with $X \in \operatorname{ker} \boldsymbol{A}$ and some $W \in T M$. Let us prove that for each $Z \in \operatorname{ker} \boldsymbol{A} \boldsymbol{B}$, the vector field $\boldsymbol{A} W \in \operatorname{ker} \boldsymbol{B}$. In fact, taking into account that $\boldsymbol{A}$ and $\boldsymbol{B}$ commute, the condition $\boldsymbol{A} \boldsymbol{B} Z=\mathbf{0}$ implies that $\boldsymbol{B} \boldsymbol{A}^{2} W=\mathbf{0}$, i.e., $\boldsymbol{B} W \in \operatorname{ker} \boldsymbol{A}^{2}=\operatorname{ker} \boldsymbol{A}$. Therefore, $\boldsymbol{A} W \in \operatorname{ker} \boldsymbol{B}$.

Corollary 6. Under the same hypotheses of Lemma 5, we have

$$
\text { ker } \boldsymbol{A} \boldsymbol{B}=\operatorname{ker} \boldsymbol{A}^{2} \boldsymbol{B}^{2} .
$$

2.2. A new family of generalized Nijenhuis torsions. We shall now generalize the notion of Haantjes torsion by means of a recursive procedure. Indeed, one can introduce a "tower" of generalized torsions of Nijenhuis type.

Definition 7. We define the univariate generalized Nijenhuis torsion of level $n$ as the vector-valued 2-form given by

$$
\begin{aligned}
& \tau_{\boldsymbol{L}}^{(n)}(X, Y):=\boldsymbol{L}^{2} \tau_{\boldsymbol{L}}^{(n-1)}(X, Y)+\tau_{\boldsymbol{L}}^{(n-1)}(\boldsymbol{L} X, \boldsymbol{L} Y)-\boldsymbol{L}\left(\tau_{\boldsymbol{L}}^{(n-1)}(X, \boldsymbol{L} Y)+\tau_{\boldsymbol{L}}^{(n-1)}(\boldsymbol{L} X, Y)\right), n \geq 1 \\
& \text { where } \tau_{\boldsymbol{L}}^{(0)}(X, Y)=[X, Y], X, Y \in T M . \text { Here } \tau_{\boldsymbol{L}}^{(1)}=\tau_{\boldsymbol{L}} \text { and } \tau_{\boldsymbol{L}}^{(2)}=\mathcal{H}_{\boldsymbol{L}} .
\end{aligned}
$$

The expression of the $n$ th-level torsion in local coordinates is given by

$$
\left(\tau_{\boldsymbol{L}}^{(n)}\right)_{j k}^{i}=\sum_{\alpha, \beta=1}^{n}\left(\boldsymbol{L}_{\alpha}^{i} \boldsymbol{L}_{\beta}^{\alpha}\left(\tau_{\boldsymbol{L}}^{(n-1)}\right)_{j k}^{\beta}+\left(\tau_{\boldsymbol{L}}^{(n-1)}\right)_{\alpha \beta}^{i} \boldsymbol{L}_{j}^{\alpha} \boldsymbol{L}_{k}^{\beta}-\boldsymbol{L}_{\alpha}^{i}\left(\left(\tau_{\boldsymbol{L}}^{(n-1)}\right)_{\beta k}^{\alpha} \boldsymbol{L}_{j}^{\beta}+\left(\tau_{\boldsymbol{L}}^{(n-1)}\right)_{j \beta}^{\alpha} \boldsymbol{L}_{k}^{\beta}\right)\right) .
$$

We wish to recall that a notion of generalized Nijenhuis torsions was also proposed, in a independent way, in [18. In that algebraic construction, one considers generalized torsions of order $n$ associated more generally to an arbitrary vector-valued skew symmetric bilinear map on a real vector space. In our geometric approach, which is a recursive one, we work on a tangent bundle and fix the initial condition of the recurrence with the standard choice of the Lie bracket between vector fields.

2.3. General properties of Haantjes operators. We shall first consider some specific cases, in which the construction of the Nijenhuis and Haantjes torsions is particularly simple.

Example 8. Let $\boldsymbol{L}: T M \rightarrow T M$ be an operator field that takes the diagonal form

$$
\boldsymbol{L}(\boldsymbol{x})=\sum_{i=1}^{n} l_{i}(\boldsymbol{x}) \frac{\partial}{\partial x^{i}} \otimes \mathrm{d} x^{i}
$$


in some local chart $\boldsymbol{x}=\left(x^{1}, \ldots, x^{n}\right)$. The components of its Nijenhuis torsion read

$$
\left(\mathcal{T}_{\boldsymbol{L}}\right)_{j k}^{i}=\left(l_{j}-l_{k}\right)\left(\frac{\partial l_{j}}{\partial x^{k}} \delta_{j}^{i}+\frac{\partial l_{k}}{\partial x^{j}} \delta_{k}^{i}\right) .
$$

It is evident that $\left(\mathcal{T}_{\boldsymbol{L}}\right)_{j k}^{i}=0$ if $i, j$ and $k$ are all distinct or if $j=k$. Thus, we can limit ourselves to analyze the $n(n-1)$ components

$$
\left(\mathcal{T}_{\boldsymbol{L}}\right)_{j k}^{j}=\left(l_{j}-l_{k}\right) \frac{\partial l_{j}}{\partial x^{k}}, \quad j \neq k
$$

If $\frac{\partial l_{j}}{\partial x^{k}} \neq 0$, each component vanishes if and only if $l_{j}(\boldsymbol{x}) \equiv l_{k}(\boldsymbol{x})$. Therefore, we can state the following

Lemma 9. Let $\boldsymbol{L}$ be the diagonal operator field (15), and assume that its Nijenhuis torsion vanishes. Let us denote by $\left(i_{1}, \ldots, i_{j}, \ldots, i_{r}\right), r \leq n$ an ordered subset of $(1,2, \ldots, n)$. If the $i_{j}$ th eigenvalue of $\boldsymbol{L}$ depends on the variables $\left(i_{1}, \ldots, i_{j}, \ldots, i_{r}\right)$, then

$$
l_{i_{j}}\left(x^{i_{1}}, \ldots, x^{i_{j}}, \ldots, x^{i_{r}}\right)=l_{i_{1}}=l_{i_{2}}=\ldots=l_{i_{r}} .
$$

In addition to the trivial case when each eigenvalue is constant, we can distinguish several further cases, in which the Nijenhuis torsion of a diagonal operator vanishes. For instance,

(i) $l_{j}(\boldsymbol{x})=\lambda_{j}\left(x^{j}\right)$

$j=1, \ldots, n \Longrightarrow n$ simple eigenvalues

(ii) $l_{j}(\boldsymbol{x})=\lambda(\boldsymbol{x})$

$j=1, \ldots, n \Longrightarrow 1$ eigenvalue of multiplicity $n$

An exhaustive analysis of all the remaining, intermediate possibilities is left to the reader.

Example 10. Let $\operatorname{dim} M=2$. It is easy to prove by a straightforward computation that the Haantjes torsion of any field of smooth operators vanishes.

Example 11. Let $\boldsymbol{L}$ be the diagonal operator of Eq. (15). The components of its Haantjes torsion read

$$
\left(\mathcal{H}_{\boldsymbol{L}}\right)_{j k}^{i}=\left(l_{i}-l_{j}\right)\left(l_{i}-l_{k}\right)\left(\mathcal{T}_{\boldsymbol{L}}\right)_{j k}^{i}=0,
$$

where $\left(\mathcal{T}_{\boldsymbol{L}}\right)_{j k}^{i}$ is given by Eq. (16).

Proposition 12. Let $\boldsymbol{L}: T M \rightarrow T M$ be an operator field. If there exists a local coordinate chart $\left(x^{1}, \ldots, x^{n}\right)$ where $\boldsymbol{L}$ takes the diagonal form (15), then the Haantjes torsion of $\boldsymbol{L}$ identically vanishes.

Due to the relevance of the Haantjes (Nijenhuis) vanishing condition, we propose the following

Definition 13. A Haantjes (Nijenhuis) operator is an operator field whose Haantjes (Nijenhuis) torsion identically vanishes.

We also recall that the transposed operator $\boldsymbol{L}^{T}: T^{*} M \mapsto T^{*} M$ is defined to be the transposed linear map of $\boldsymbol{L}$ with respect to the natural pairing between the tangent and the cotangent bundle of $M$ :

$$
\left\langle\boldsymbol{L}^{T} \alpha, X\right\rangle=\langle\alpha, \boldsymbol{L} X\rangle \quad \alpha \in T^{*} M, X \in T M .
$$


The condition for a Nijenhuis operator $\boldsymbol{N}$ to be torsionless can be expressed in terms of its Lie derivative along the flow of any vector field $X \in T M$ in the following, equivalent way (see, for instance, [13]):

$$
\mathcal{L}_{N X}(\boldsymbol{N})=\boldsymbol{N} \mathcal{L}_{X}(\boldsymbol{N}) .
$$

Analogously, the vanishing of the Haantjes torsion (3) of an operator field $\boldsymbol{L}$ is equivalent to the novel condition

$$
\mathcal{L}_{\boldsymbol{L}^{2} X}(\boldsymbol{L}) \boldsymbol{L}=\boldsymbol{L}^{3} \mathcal{L}_{X}(\boldsymbol{L})-\boldsymbol{L}^{2}\left(2 \mathcal{L}_{\boldsymbol{L} X}(\boldsymbol{L})+\mathcal{L}_{X}(\boldsymbol{L}) \boldsymbol{L}\right)+\boldsymbol{L}\left(\mathcal{L}_{\boldsymbol{L}^{2} X}(\boldsymbol{L})+2 \mathcal{L}_{\boldsymbol{L} X}(\boldsymbol{L}) \boldsymbol{L}\right) .
$$

As is well known (see for instance [13]), given an invertible Nijenhuis operator, its inverse is also a Nijenhuis operator. The same property holds true for a Haantjes operator.

Proposition 14. Let $\boldsymbol{L}: T M \rightarrow T M$ be a Haantjes operator. If $\boldsymbol{L}^{-1}$ exists, it is also a Haantjes operator.

Proof. It is a consequence of the identity

$$
\mathcal{H}_{\boldsymbol{L}^{-1}}(X, Y)=\boldsymbol{L}^{-4} \mathcal{H}_{\boldsymbol{L}}\left(\boldsymbol{L}^{-2} X, \boldsymbol{L}^{-2} Y\right)
$$

that can be easily deduced from Eq. (4). For an alternative proof, see Proposition 2, p. 257 of [4].

The product of a Nijenhuis operator with a generic function is no longer a Nijenhuis operator, as is proved by the identity

$\mathcal{T}_{f \boldsymbol{L}}(X, Y)=f^{2} \mathcal{T}_{\boldsymbol{L}}(X, Y)+f\left((\boldsymbol{L} X)(f) \boldsymbol{L} Y-(\boldsymbol{L} Y)(f) \boldsymbol{L} X+Y(f) \boldsymbol{L}^{2} X-X(f) \boldsymbol{L}^{2} Y\right)$,

where $X(f)$ denotes the Lie derivative of an arbitrary function $f \in C^{\infty}(M)$ with respect to the vector field $X$. However, the differential and algebraic properties of the Haantjes operators are much richer, as one can infer from the following, remarkable results.

Proposition 15 (4]). Let $\boldsymbol{L}: T M \rightarrow T M$ be an operator field, $f, g: M \rightarrow \mathbb{R}$ two $C^{\infty}(M)$ functions, and $\boldsymbol{I}$ the identity operator in $T M$. Then we have

$$
\mathcal{H}_{f \boldsymbol{I}+g \boldsymbol{L}}(X, Y)=g^{4} \mathcal{H}_{\boldsymbol{L}}(X, Y) .
$$

Proof. See Proposition 1, p. 255 of [4].

Proposition 16 ([5]). Let $\boldsymbol{L}: T M \rightarrow T M$ be a Haantjes operator. Then, for any polynomial in $\boldsymbol{L}$ with coefficients $a_{j} \in C^{\infty}(M)$, the associated Haantjes torsion vanishes, i.e.

$$
\mathcal{H}_{\boldsymbol{L}}(X, Y)=\mathbf{0} \Longrightarrow \mathcal{H}_{\left(\sum_{j} a_{j}(\boldsymbol{x}) \boldsymbol{L}^{j}\right)}(X, Y)=\mathbf{0} .
$$

Proof. See Corollary 3.3, p. 1136 of [5].

As we shall see in Section 5.2, as a consequence of Propositions 15] and 16, it follows that a Haantjes operator generates a cyclic Haantjes algebra (i. e. a cyclic algebra of Haantjes operators) over the ring of smooth functions on $M$. However, this is not the case for a Nijenhuis operator $\boldsymbol{N}$, since a polynomial in $\boldsymbol{N}$ with coefficients $a_{j} \in C^{\infty}(M)$ is not necessarily a Nijenhuis operator. 
2.4. An application to Classical Mechanics: the inertia tensor. We wish to present a new example of application of Nijenhuis and Haantjes operators, borrowed from Classical Mechanics.

Example 17. Let $\mathcal{M}=\left\{\left(P_{\gamma}, m_{\gamma}\right) \in\left(\mathcal{E}_{n}, \mathbb{R}\right)\right\}$ be a finite system of mass points (possibly with $m_{\gamma}<0$ ) in the $n$-dimensional affine Euclidean space $\mathcal{E}_{n}$. Let us consider the $(1,1)$ tensor field defined by

$$
\boldsymbol{E}_{P}(\vec{v})=\sum_{\gamma} m_{\gamma}\left(\left(P_{\gamma}-P\right) \cdot \vec{v}\right)\left(P_{\gamma}-P\right) \quad \vec{v} \in T_{P} \mathcal{E}_{n} \equiv \mathbb{E}_{n}
$$

known as the planar inertia tensor (or Euler tensor in Continuum Mechanics). The inertia tensor is given by

$$
\mathbb{I}_{P}(\vec{v})=\sum_{\gamma} m_{\gamma}\left(\left|P_{\gamma}-P\right|^{2} \vec{v}-\left(\left(P_{\gamma}-P\right) \cdot \vec{v}\right)\left(P_{\gamma}-P\right)\right) .
$$

They are related by the formulae

$$
\mathbb{I}_{P}=\operatorname{Trace}\left(\boldsymbol{E}_{P}\right) \boldsymbol{I}_{n}-\boldsymbol{E}_{P}, \quad \boldsymbol{E}_{P}=\frac{\operatorname{Trace}\left(\mathbb{I}_{P}\right)}{n-1} \boldsymbol{I}_{n}-\mathbb{I}_{P}, \quad n>1,
$$

where $\boldsymbol{I}_{n}$ is the identity operator in $\mathbb{E}_{n}$. Both of them are symmetric w.r.t. the Euclidean scalar product, so that they are diagonalizable at any point of $\mathcal{E}_{n}$. Furthermore, by virtue of relations (28) they commute; consequently, they can be pointwise diagonalized, simultaneously.

If $G$ is the center of mass of $\mathcal{M}$, defined by

$$
G-P=\frac{1}{m} \sum_{\gamma}\left(P_{\gamma}-P\right) \quad m:=\sum_{\gamma} m_{\gamma} \quad m \in \mathbb{R} \backslash\{0\},
$$

the following Huygens-Steiner transposition formulae hold

$$
\begin{aligned}
\boldsymbol{E}_{P}(\vec{v}) & =\boldsymbol{E}_{G}(\vec{v})+m((P-G) \cdot \vec{v})(P-G), \\
\mathbb{I}_{P}(\vec{v}) & =\mathbb{I}_{G}(\vec{v})+m|P-G|^{2}-m((P-G) \cdot \vec{v})(P-G) .
\end{aligned}
$$

From Eqs. (29) and (30) it follows that in the Cartesian coordinates $\left(x^{1}, \ldots, x^{n}\right)$ with origin in $G$, defined by the common eigen-directions of $E_{G}$ and $\mathbb{I}_{G}$, we have

$$
\begin{aligned}
\left(\boldsymbol{E}_{P}\right)_{j}^{i} & =\lambda_{i}(G) \delta_{j}^{i}+m x^{i} x_{j}, \\
\left(\mathbb{I}_{P}\right)_{j}^{i} & =l_{i}(G) \delta_{j}^{i}+m\left(\sum_{\alpha=1}^{n} x^{\alpha} x_{\alpha}-x^{i} x_{j}\right) \quad i, j=1, \ldots, n .
\end{aligned}
$$

Here $x_{\alpha}=\delta_{\alpha \beta} x^{\beta}$, and $\lambda_{i}(G)$ and $l_{j}(G)$ denote the eigenvalues of the tensor fields $\boldsymbol{E}$ and II respectively, both evaluated at the point $G$. In [1, 2] it has been proved that the Nijenhuis torsion of $\boldsymbol{E}$ vanishes

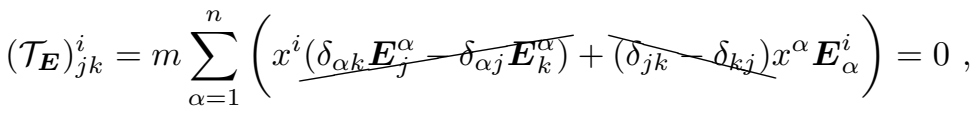

thus its Haantjes torsion also vanishes. Furthermore, we observe that

$$
\left(\mathcal{T}_{\mathbb{I}}\right)_{j k}^{i}=2 m \sum_{\alpha=1}^{n}\left(x_{\alpha}\left(\mathbb{I}_{j}^{\alpha} \delta_{k}^{i}-\mathbb{I}_{k}^{\alpha} \delta_{j}^{i}\right)+x_{k} \mathbb{I}_{j}^{i}-x_{j} \mathbb{I}_{k}^{i}\right),
$$

i.e. the Nijenhuis torsion of $\mathbb{I}$ is not identically zero, although its Haantjes torsion vanishes as a consequence of the identity (24), applied to the first equation (28). 
Thus, the planar inertia tensor $\boldsymbol{E}_{P}(\vec{v})$ is a Nijenhuis operator, whereas the inertia tensor $\mathbb{I}_{P}(\vec{v})$ is a Haantjes operator.

Other relevant examples of Haantjes operators expressed in terms of Killing tensors in a Riemannian manifold can be found in [32.

\section{The GeOMEtry of HaAntJes operators}

3.1. Integrability. As stated in Proposition 12 the Haantjes torsion $\mathcal{H}_{\boldsymbol{L}}$ of a semisimple operator field $\boldsymbol{L}$ has a relevant geometrical meaning: its vanishing is a necessary condition for the eigen-distributions of $\boldsymbol{L}$ to be integrable. To clarify this point, first we need to recall that a reference frame is a set of $n$ vector fields $\left\{Y_{1}, \ldots, Y_{n}\right\}$ such that, at each point $\boldsymbol{x} \in U \subseteq M, U$ open set, they form a basis of the tangent space $T_{\boldsymbol{x}} U$. Two frames $\left\{X_{1}, \ldots, X_{n}\right\}$ and $\left\{Y_{1}, \ldots, Y_{n}\right\}$ are said to be equivalent if $n$ nowhere vanishing smooth functions $f_{i}$ exist, such that

$$
X_{i}=f_{i}(\boldsymbol{x}) Y_{i}, \quad i=1, \ldots, n .
$$

A natural frame is the frame associated to a local chart $\left\{U,\left(x^{1}, \ldots, x^{n}\right)\right\}$ which is formed by the vector fields $\left\{\frac{\partial}{\partial x^{1}}, \ldots, \frac{\partial}{\partial x^{n}}\right\}$.

Definition 18. An integrable frame is a reference frame equivalent to a natural frame.

Proposition 19 ([3]). A reference frame $\left\{Y_{1}, \ldots, Y_{n}\right\}$ on a manifold $M$ is integrable if and only one the following two, equivalent conditions are satisfied:

- each distribution generated by any two vector fields $\left\{Y_{i}, Y_{j}\right\}$ is Frobenius integrable;

- each distribution $\mathcal{E}_{i}$ generated by all the vector fields except $Y_{i}$ is Frobenius integrable.

Definition 20. An operator field $\boldsymbol{L}$ is semisimple if, in each open neighborhood $U \subseteq M$, there exists a reference frame formed by proper eigenvector fields of $\boldsymbol{L}$.

This frame will be said to be an eigen-frame of $\boldsymbol{L}$. Moreover, $\boldsymbol{L}$ is simple if all of its eigenvalues are pointwise distinct, namely $l_{i}(\boldsymbol{x}) \neq l_{j}(\boldsymbol{x}), i, j=1, \ldots, n, \forall \boldsymbol{x} \in M$.

An important problem is to establish the conditions ensuring that the eigenframes of $\boldsymbol{L}$ are integrable. Proposition 12 amounts to say that if an operator admits a local chart in which it takes a diagonal form, then its Haantjes torsion necessarily vanishes. Therefore, the associated natural frame is an eigen-frame (trivially) integrable. In 1955, Haantjes proved in 15 that the vanishing of the Haantjes torsion of a semisimple (with possibly coinciding eigenvalues) operator $\boldsymbol{L}$ is also a sufficient condition to ensure the integrability of each of its eigendistributions and their direct sums; consequently, it also guarantees the existence of local coordinate charts in which $\boldsymbol{L}$ takes a diagonal form. We shall say that such coordinates are a set of Haantjes coordinates for $\boldsymbol{L}$. We remark that, in the framework of hydrodynamic systems, when the eigenvalues are simple, the Haantjes coordinates coincide with the Riemann invariants of the system. Furthermore, Haantjes stated that the vanishing of the Haantjes torsion of an operator with real eigenvalues $\boldsymbol{L}$ is also a sufficient (but not necessary) condition to ensure the integrability of each of its generalized eigen-distributions (of constant rank) and their direct sums. An equivalent statement of the above-mentioned results is that each 
Haantjes operator with real eigenvalues admits an integrable frame of generalized eigenvector fields.

Below, we prove necessary and sufficient conditions for the integrability of generalized eigen-distributions of non-semisimple operator fields. Let us denote by $\operatorname{Spec}(\boldsymbol{L}):=\left\{l_{1}(\boldsymbol{x}), l_{2}(\boldsymbol{x}), \ldots, l_{s}(\boldsymbol{x})\right\}$ the set of the eigenvalues of an operator $\boldsymbol{L}$, which we shall always assume to be real and pointwise distinct in all the forthcoming considerations. Also, we denote by

$$
\mathcal{D}_{i}=\operatorname{ker}\left(\boldsymbol{L}-l_{i} \boldsymbol{I}\right)^{\rho_{i}}, \quad i=1, \ldots, s
$$

the $i$ th generalized eigen-distribution, that is the distribution of all the generalized eigenvector fields corresponding to the eigenvalue $l_{i}=l_{i}(\boldsymbol{x})$. In Eq. (35), $\rho_{i}$ stands for the Riesz index of $l_{i}$ (or equivalently, the index of the associated eigendistribution), defined as the minimum integer such that

$$
\operatorname{ker}\left(\boldsymbol{L}-l_{i} \boldsymbol{I}\right)^{\rho_{i}}=\operatorname{ker}\left(\boldsymbol{L}-l_{i} \boldsymbol{I}\right)^{\rho_{i}+1},
$$

which we shall always assume to be independent of $\boldsymbol{x}$ (the Riesz index of $\boldsymbol{L}$, introduced in Lemma 4, corresponds to the case $l_{i}=0$ ). When $\rho_{i}=1, \mathcal{D}_{i}$ is a proper eigen-distribution.

Definition 21. A generalized eigen-frame of an operator field $\boldsymbol{L}$ is a local reference frame of generalized eigenvector fields of $\boldsymbol{L}$.

Let us recall that the tangent space at any point of $\boldsymbol{x} \in M$ admits the spectral decomposition

$$
T_{\boldsymbol{x}} M=\bigoplus_{i=1}^{s} \mathcal{D}_{i}(\boldsymbol{x})
$$

Theorem 22. Let $\boldsymbol{L}: T M \rightarrow T M$ be an operator field and $\mathcal{D}_{i}$ one of its generalized eigen-distributions, with Riesz index $\rho_{i}, i \in\{1, \ldots, s\}$. We assume that the rank of $\mathcal{D}_{i}$ is independent of $\boldsymbol{x} \in M$. Then, the following three conditions are equivalent:

(1) The distribution $\mathcal{D}_{i}$ is involutive;

(2) $\tau_{\left(\boldsymbol{L}-l_{i} \boldsymbol{I}\right)^{\rho_{i}}}\left(\mathcal{D}_{i}, \mathcal{D}_{i}\right)=\mathbf{0}$;

(3) $\mathcal{H}_{\left(\boldsymbol{L}-l_{i} \boldsymbol{I}\right)^{\rho_{i}}}\left(\mathcal{D}_{i}, \mathcal{D}_{i}\right)=\mathbf{0}$

Proof. First, let us prove that (1) $\Leftrightarrow(2)$. From Eqs. (35) and (7) we obtain

$$
\tau_{\left(\boldsymbol{L}-l_{i} \boldsymbol{I}\right)^{\rho_{i}}}\left(\mathcal{D}_{i}, \mathcal{D}_{i}\right)=\left(\boldsymbol{L}-l_{i} \boldsymbol{I}\right)^{2 \rho_{i}}\left[\mathcal{D}_{i}, \mathcal{D}_{i}\right]
$$

Therefore, we deduce that

$$
\left[\mathcal{D}_{i}, \mathcal{D}_{i}\right] \subseteq \operatorname{ker}\left(\boldsymbol{L}-l_{i} \boldsymbol{I}\right)^{2 \rho_{i}} \stackrel{\sqrt[36]{=}}{=} \operatorname{ker}\left(\boldsymbol{L}-l_{i} \boldsymbol{I}\right)^{\rho_{i}}
$$

if and only if condition (2) is fulfilled. Obviously, condition (2) implies (3). Consequently, condition (1) implies (3). Finally, let us prove that condition (3) implies (1). In fact, from Eqs. (35) and (8) it follows that

$$
\mathcal{H}_{\left(\boldsymbol{L}-l_{i} \boldsymbol{I}\right)^{\rho_{i}}}\left(\mathcal{D}_{i}, \mathcal{D}_{i}\right)=\left(\boldsymbol{L}-l_{i} \boldsymbol{I}\right)^{4 \rho_{i}}\left[\mathcal{D}_{i}, \mathcal{D}_{i}\right] .
$$

Therefore,

$$
\left[\mathcal{D}_{i}, \mathcal{D}_{i}\right] \subseteq \operatorname{ker}\left(\boldsymbol{L}-l_{i} \boldsymbol{I}\right)^{4 \rho_{i}} \stackrel{(36)}{=} \operatorname{ker}\left(\boldsymbol{L}-l_{i} \boldsymbol{I}\right)^{\rho_{i}}
$$

if condition (3) is fulfilled. 
Theorem 23. Let $\boldsymbol{L}: T M \rightarrow T M$ be an operator field, and $\mathcal{D}_{i}, \mathcal{D}_{j}$ two involutive eigen-distributions with Riesz indices $\rho_{i}$ and $\rho_{j}$, respectively $(i, j \in\{1, \ldots, s\})$. Then, the following three conditions are equivalent:

(1) the distribution $\mathcal{D}_{i} \oplus \mathcal{D}_{j}$ is involutive;

(2) $\tau_{\left(\boldsymbol{L}-l_{i} \boldsymbol{I}\right)^{\rho_{i}}\left(\boldsymbol{L}-l_{j} \boldsymbol{I}\right)^{\rho_{j}}}\left(\mathcal{D}_{i} \oplus \mathcal{D}_{j}, \mathcal{D}_{i} \oplus \mathcal{D}_{j}\right)=\mathbf{0}$;

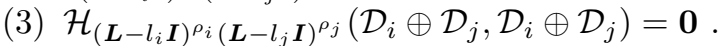

Proof. Firstly, let us prove that (1) $\Leftrightarrow(2)$. From Eq. (7) applied to $\boldsymbol{A}:=\left(\boldsymbol{L}-l_{i} \boldsymbol{I}\right)^{\rho_{i}}$, $\boldsymbol{B}:=\left(\boldsymbol{L}-l_{j} \boldsymbol{I}\right)^{\rho_{j}}$ and (11) it follows that

$$
\tau_{\boldsymbol{A} \boldsymbol{B}}\left(\mathcal{D}_{i} \oplus \mathcal{D}_{j}, \mathcal{D}_{i} \oplus \mathcal{D}_{j}\right)=\boldsymbol{A}^{2} \boldsymbol{B}^{2}\left[\mathcal{D}_{i} \oplus \mathcal{D}_{j}, \mathcal{D}_{i} \oplus \mathcal{D}_{j}\right]=\boldsymbol{A}^{2} \boldsymbol{B}^{2}\left[\mathcal{D}_{i}, \mathcal{D}_{j}\right]
$$

Therefore,

$$
\left[\mathcal{D}_{i}, \mathcal{D}_{j}\right] \subseteq \operatorname{ker} \boldsymbol{A}^{2} \boldsymbol{B}^{2} \stackrel{[36],(12)}{=} \operatorname{ker} \boldsymbol{A} \boldsymbol{B} \stackrel{[11]}{=} \operatorname{ker} \boldsymbol{A} \oplus \operatorname{ker} \boldsymbol{B}
$$

if and only if condition (2) is fulfilled. Obviously, condition (2) implies (3), therefore (1) implies (3). Finally, from Eq. (11) and (8) it follows that

$$
\mathcal{H}_{\boldsymbol{A} \boldsymbol{B}}\left(\mathcal{D}_{i} \oplus \mathcal{D}_{j}, \mathcal{D}_{i} \oplus \mathcal{D}_{j}\right)=\boldsymbol{A}^{4} \boldsymbol{B}^{4}\left[\mathcal{D}_{i} \oplus \mathcal{D}_{j}, \mathcal{D}_{i} \oplus \mathcal{D}_{j}\right]=\boldsymbol{A}^{4} \boldsymbol{B}^{4}\left[\mathcal{D}_{i}, \mathcal{D}_{j}\right]
$$

Consequently

$$
\left[\mathcal{D}_{i}, \mathcal{D}_{j}\right] \subseteq \operatorname{ker} \boldsymbol{A}^{4} \boldsymbol{B}^{4} \stackrel{\sqrt{36]}, \sqrt{12})}{=} \operatorname{ker} \boldsymbol{A} \boldsymbol{B} \stackrel{(11)}{=} \operatorname{ker} \boldsymbol{A} \oplus \operatorname{ker} \boldsymbol{B}
$$

if condition (3) is satisfied.

\subsection{A new proof of the Haantjes theorem.}

Definition 24. Let us consider a set of distributions $\left\{\mathcal{D}_{i}, \mathcal{D}_{j}, \ldots, \mathcal{D}_{k}\right\}$. We shall say that such distributions are mutually integrable if

(i) each of them is integrable;

(ii) any sum $\mathcal{D}_{i}+\mathcal{D}_{j}+\cdots+\mathcal{D}_{k}$ (where all indices $i, j, \ldots, k$ are different) is also integrable.

Now, we are in the position to recover the Haantjes theorem as a consequence of Theorems 22 and 23 .

Theorem 25 (15]). Let $\boldsymbol{L}: T M \rightarrow T M$ be an operator field, and assume that the rank of each generalized eigen-distribution $\mathcal{D}_{i}, i=1, \ldots, s$ is independent of $\boldsymbol{x} \in M$. The vanishing of the Haantjes torsion

$$
\mathcal{H}_{\boldsymbol{L}}(X, Y)=\mathbf{0} \quad \forall X, Y \in T M
$$

is a sufficient condition to ensure the mutual integrability of the generalized eigendistributions $\left\{\mathcal{D}_{1}, \ldots, \mathcal{D}_{s}\right\}$. In addition, if $\boldsymbol{L}$ is semisimple, condition (38) is also necessary.

Proof. In the non-semisimple case, according to Proposition 16, if condition (38) is satisfied, then the conditions (3) of Theorems 22 and 23 hold for $i=1, \ldots, s$; therefore, the distributions $\left\{\mathcal{D}_{1}, \ldots, \mathcal{D}_{s}\right\}$ are mutually integrable.

Conversely, if the distributions $\left\{\mathcal{D}_{1}, \ldots, \mathcal{D}_{s}\right\}$ are mutually integrable, then the conditions (3) of Theorems 22 and 23 are satisfied for all eigen-distributions $\mathcal{D}_{i}$. In addition, if $\boldsymbol{L}$ is semisimple, then each Riesz index $\rho_{i}=1$, and Proposition 15 implies that

$$
\mathcal{H}_{\boldsymbol{L}-l_{i} \boldsymbol{I}}(X, Y)=\mathcal{H}_{\boldsymbol{L}}(X, Y), \quad \forall X, Y \in T M, \quad i=1, \ldots, s .
$$


Therefore, due to the spectral decomposition (37), we deduce that condition (38) is fulfilled.

Remark 26. Due to Theorems 20 and 23, conditions (2) are not stronger than conditions (3) but equivalent. This can be understood by observing that if a distribution $\mathcal{D}_{i}$ is integrable, then the operator $\left(\boldsymbol{L}-l_{i} \boldsymbol{I}\right)^{\rho_{i}}$ can be restricted to each integral leaf of $\mathcal{D}_{i}$ and such restriction vanishes. Thus, one might wonder, at least in the semisimple case, whether there exists an analogous of Proposition 15 for the Nijenhuis torsion. This is not the case as, unlike Eq. (39), we have

$$
\tau_{\boldsymbol{L}-l_{i} \boldsymbol{I}}(X, Y)=\tau_{\boldsymbol{L}}(X, Y)+(\boldsymbol{L} Y)\left(l_{i}\right) X-(\boldsymbol{L} X)\left(l_{i}\right) Y,
$$

which does not coincide with $\tau_{\boldsymbol{L}}(X, Y)$ unless the eigenvalue $l_{i}$ is constant.

In the article by Haantjes [15], the original proof of Theorem 25] which was developed in a completely different way, is explicitly carried out only for the case of a semisimple operator. In [9], the integrability of the eigen-distributions of a Nijenhuis operator with generalized eigenvectors of Riesz index 2 was proved. However, the case of Haantjes operators was not considered. Besides, to the best of our knowledge, the proofs of the Haantjes theorem available in the literature (see for instance [12, 13]) are based on the quite restrictive assumption that the Haantjes operator is semisimple.

Let us show in detail how we can determine a coordinate system that, under the assumptions of Theorem 25, allows us to write a Haantjes operator $\boldsymbol{L}$ in a block-diagonal form. Denote by

$$
\mathcal{E}_{i}:=\operatorname{Im}\left(\boldsymbol{L}-l_{i} \mathbf{I}\right)^{\rho_{i}}=\bigoplus_{j=1, j \neq i}^{s} \mathcal{D}_{j}, \quad i=1, \ldots, s
$$

the distribution of corank $r_{i}$ (being $r_{i}$ the rank of $\mathcal{D}_{i}$ ), spanned by all the generalized eigenvectors of $\boldsymbol{L}$, except those associated with the eigenvalue $l_{i}$. Such a distribution will be said to be a characteristic distribution of $\boldsymbol{L}$. Let $\mathcal{E}_{i}^{\circ}$ denote the annihilator of the distribution $\mathcal{E}_{i}$. Observe that, since $\boldsymbol{L}$ by hypothesis has real eigenvalues, the cotangent spaces of $M$ can be locally decomposed as

$$
T_{\boldsymbol{x}}^{*} M=\bigoplus_{i=1}^{s} \mathcal{E}_{i}^{\circ}(\boldsymbol{x})
$$

Moreover, each characteristic distribution $\mathcal{E}_{i}$ is integrable by virtue of Theorem 25. We shall denote by $\mathrm{E}_{i}$ the foliation associated with $\mathcal{E}_{i}$ and by $E_{i}(\boldsymbol{x})$ the connected leave through $\boldsymbol{x}$ belonging to $\mathrm{E}_{i}$. Thus, the set of distributions $\left\{\mathcal{E}_{1}, \mathcal{E}_{2}, \ldots, \mathcal{E}_{s}\right\}$ generates as many foliations $\left\{\mathrm{E}_{1}, \mathrm{E}_{2}, \ldots, \mathrm{E}_{s}\right\}$ as the number of distinct eigenvalues of $\boldsymbol{L}$. This set of foliations will be referred to as the characteristic web of $\boldsymbol{L}$ and the leaves $E_{i}(\boldsymbol{x})$ of each foliation $\mathrm{E}_{i}$ as the characteristic fibers of the web.

Definition 27. A collection of $r_{i}$ smooth functions will be said to be adapted to a foliation $E_{i}$ of the characteristic web of a Haantjes operator $\boldsymbol{L}$ if the level sets of such functions coincide with the characteristic fibers of the foliation $E_{i}$.

Definition 28. A parametrization of the characteristic web of a Haantjes operator $\boldsymbol{L}$ is an ordered set of $n$ independent smooth functions grouped as $\left(\boldsymbol{f}^{1}, \ldots, \boldsymbol{f}^{i}, \ldots, \boldsymbol{f}^{s}\right)$ such that for any $i=1, \ldots, s$, the ordered subset $\boldsymbol{f}^{i}=\left(f^{i, 1}, \ldots, f^{i, r_{i}}\right)$ is adapted to 
the ith characteristic foliation of the web:

$$
f_{\mid E_{i}(\mathbf{x})}^{i, k}=c^{i, k} \quad \forall E_{i}(\mathbf{x}) \in \mathrm{E}_{i}, \quad k=1, \ldots, r_{i},
$$

where $c^{i, k}$ are real constants depending on the indices $i$ and $k$ only. In this case, we shall say that the collection of all these functions is adapted to the web and that each of them is a characteristic function.

Proposition 29. The vanishing of the Haantjes torsion of an operator field $\boldsymbol{L}$ is a sufficient condition to ensure that $\boldsymbol{L}$ admits an equivalence class of integrable generalized eigen-frames, where $\boldsymbol{L}$ takes a block-diagonal form. Furthermore, if $\boldsymbol{L}$ is semisimple, the condition is also necessary and $\boldsymbol{L}$ takes a diagonal form. In addition, if $\boldsymbol{L}$ is simple each of its eigen-frames is integrable.

Proof. Each characteristic distribution $\mathcal{E}_{i}$ is integrable, by virtue of the Haantjes Theorem 25. Therefore, in the corresponding annihilator $\mathcal{E}_{i}^{\circ}$ one can find $r_{i}$ exact one-forms $\left(\mathrm{d} x^{i, 1}, \ldots, \mathrm{d} x^{i, r_{i}}\right)$ that provide us with functions $\boldsymbol{x}^{i}=\left(x^{i, 1}, \ldots, x^{i, r_{i}}\right)$ adapted to the characteristic foliation $\mathrm{E}_{i}$. Collecting together all these functions, one can construct a set of $n$ independent coordinates, that we group as $\left(\boldsymbol{x}^{1}, \ldots, \boldsymbol{x}^{i}, \ldots, \boldsymbol{x}^{s}\right)$ and consequently, a local chart $\left\{U,\left(\boldsymbol{x}^{1}, \ldots, \boldsymbol{x}^{i}, \ldots, \boldsymbol{x}^{s}\right)\right\}$, adapted to the characteristic web. The corresponding natural frame $\left\{\frac{\partial}{\partial \boldsymbol{x}^{1}}, \ldots, \frac{\partial}{\partial \boldsymbol{x}^{i}}, \ldots, \frac{\partial}{\partial \boldsymbol{x}^{s}}\right\}$ turns out to be a generalized eigen-frame. In fact, as

$$
\mathcal{D}_{i}^{\circ}=\bigoplus_{j=1, j \neq i}^{s} \mathcal{E}_{j}^{\circ}
$$

any generalized eigenvector $W \in \mathcal{D}_{i}$ leaves invariant all the coordinate functions except at most the characteristic functions $\boldsymbol{x}^{i}=\left(x^{i, 1}, \ldots, x^{i, r_{i}}\right)$ of $\mathrm{E}_{i}$. Thus, we have that $W=W\left(\boldsymbol{x}^{i}\right) \frac{\partial}{\partial \boldsymbol{x}^{i}}=\sum_{k=1}^{r_{i}} W\left(x^{i, k}\right) \frac{\partial}{\partial x^{i, k}}$, therefore

$$
\mathcal{D}_{i_{\mid U}}=\left\langle\frac{\partial}{\partial x^{i, 1}}, \ldots, \frac{\partial}{\partial x^{i, r_{i}}}\right\rangle
$$

(hereafter the symbol \langle\rangle denotes the $C^{\infty}(M)$-linear span of the considered vector fields). Thus, each frame equivalent to $\left\{\frac{\partial}{\partial \boldsymbol{x}^{1}}, \ldots, \frac{\partial}{\partial \boldsymbol{x}^{i}}, \ldots, \frac{\partial}{\partial \boldsymbol{x}^{s}}\right\}$ is an integrable eigen-frame of generalized eigenvector fields. Consequently, there exists an equivalence class of integrable frames and associated local charts where the operator $\boldsymbol{L}$ takes a block-diagonal form due to the invariance of its eigen-distributions. Moreover, if $\boldsymbol{L}$ is semisimple, then its generalized eigen-frames are proper eigen-frames and the block-diagonal form reduces to a diagonal one.

Conversely, if there exists a local chart where $\boldsymbol{L}$ takes a diagonal form, then the corresponding natural frame is obviously integrable. Thus, due to Proposition 12 the Haantjes torsion of $\boldsymbol{L}$ vanishes. Finally, if $\boldsymbol{L}$ is simple, each eigen-distribution has rank 1; therefore, each natural eigen-frame fulfills the conditions of Proposition 19. The last statement is equivalent to the celebrated result that A. Nijenhuis published in 1951 [27].

Definition 30. Let $\boldsymbol{L}: T M \rightarrow T M$ be a Haantjes operator. A local chart $\left\{U,\left(x^{1}, \ldots, x^{n}\right)\right\}$ whose natural frame is a generalized eigen-frame will be said to be a Haantjes chart for $\boldsymbol{L}$. 
A Haantjes chart for $\boldsymbol{L}$ can also be computed by using the transposed operator $\boldsymbol{L}^{T}$. Let us denote by

$$
\operatorname{ker}\left(\boldsymbol{L}^{T}-l_{i} \boldsymbol{I}\right)^{\rho_{i}}
$$

the $i$ th distribution of the generalized eigen 1-forms associated with the eigenvalue $l_{i}(\boldsymbol{x})$, which fulfills the property

$$
\operatorname{ker}\left(\boldsymbol{L}^{T}-l_{i} \boldsymbol{I}\right)^{\rho_{i}}=\left(\operatorname{Im}\left(\boldsymbol{L}-l_{i} \mathbf{I}\right)^{\rho_{i}}\right)^{\circ}=\mathcal{E}_{i}^{\circ} .
$$

Such a property implies that each generalized eigenform of $\boldsymbol{L}^{T}$ annihilates all generalized eigenvectors of $\boldsymbol{L}$ associated with different eigenvalues. Moreover, it allows us to prove an interesting equivalence result.

Proposition 31. Let $\boldsymbol{L}: T M \rightarrow T M$ be a Haantjes operator. The differentials of the characteristic coordinate functions are exact generalized eigenforms for the transposed operator $\boldsymbol{L}^{T}$. Conversely, each (locally) exact generalized eigenform of $\boldsymbol{L}^{T}$ provides us with a characteristic function for the Haantjes web of $\boldsymbol{L}$.

The characteristic functions of a Haantjes operator are characterized by the following, simple property.

Proposition 32. A function $h \in C^{\infty}(M)$ is a characteristic function of a Haantjes operator $\boldsymbol{L}$, associated with its eigenvalue $l_{i}$, if and only if given a set of local coordinates adapted to the characteristic web $\left(\boldsymbol{x}^{1}, \ldots, \boldsymbol{x}^{i}, \ldots, \boldsymbol{x}^{s}\right), h$ depends, at most, on the subset of coordinates $\boldsymbol{x}^{i}=\left(x^{i, 1}, \ldots, x^{i, r_{i}}\right)$ that are constant over the leaves of the foliation $\mathrm{E}_{i}$.

Proof. If $h=h\left(x^{i, 1}, \ldots, x^{i, r_{i}}\right)$, it is constant on the leaves of $\mathrm{E}_{i}$, then $\mathrm{d} h \in \mathcal{E}_{i}^{\circ}$. Vice versa, if we assume that $\mathrm{d} h \in \mathcal{E}_{i}^{\circ}$, then it can be expressed in terms of a linear combination (with functions as coefficients) of $\left\{\mathrm{d} x^{i, 1}, \ldots, \mathrm{d} x^{i, r_{i}}\right\}$ only. The result follows by observing that $\mathrm{d} h$ is an exact 1 -form.

\section{Characteristic HaAntjes Coordinates and Block Diagonalization}

We shall present below some of our main results, concerning the existence of charts of coordinates in which a family of commuting Haantjes operators takes a block-diagonal form.

Proposition 33. Let $\mathcal{K}=\left\{\boldsymbol{K}_{1}, \ldots, \boldsymbol{K}_{w}\right\}, \boldsymbol{K}_{\alpha}: T M \rightarrow T M, \alpha=1, \ldots, w$ be a family of commuting operator fields; we assume that one of them, say $\boldsymbol{K}_{1}$, has vanishing Haantjes torsion. Then, there exist local charts in which all of the operators $\boldsymbol{K}_{\alpha}$ can be written simultaneously in a block-diagonal form. In addition, if we assume that

(i) all the operators of the family have vanishing Haantjes torsion,

(ii) all possible nontrivial intersections of their generalized eigen-distributions

$$
\mathcal{V}_{a}(\boldsymbol{x}):=\bigoplus_{i_{1}, \ldots, i_{w}}^{s_{1}, \ldots, s_{w}} \mathcal{D}_{i_{1}}^{(1)}(\boldsymbol{x}) \bigcap \ldots \bigcap \mathcal{D}_{i_{w}}^{(w)}(\boldsymbol{x}), \quad a=1, \ldots, v \leq n
$$

are mutually integrable, then there exist sets of local coordinates, adapted to the decomposition

$$
T_{\boldsymbol{x}} M=\bigoplus_{a=1}^{v} \mathcal{V}_{a}(\boldsymbol{x}) \quad \boldsymbol{x} \in M
$$


in which all operators $\boldsymbol{K}_{\alpha}$ admit simultaneously a block-diagonal form (with possibly finer blocks).

Proof. Due to Proposition 29, there exists an equivalence class of integrable frames and local charts where the Haantjes operator $\boldsymbol{K}_{1}$ takes a block-diagonal form. Such coordinates are adapted to the characteristic web associated with the spectral decomposition of $\boldsymbol{K}_{1}$ :

$$
T_{\boldsymbol{x}} M=\mathcal{D}_{i_{1}}^{(1)}(\boldsymbol{x}) \bigoplus \mathcal{E}_{i_{1}}^{(1)}(x)=\bigoplus_{i_{1}=1}^{s_{1}} \mathcal{D}_{i_{1}}^{(1)}(\boldsymbol{x})
$$

These coordinates will be denoted by

$$
\boldsymbol{x}=\left(\boldsymbol{x}^{1}, \ldots, \boldsymbol{x}^{i_{1}}, \ldots, \boldsymbol{x}^{s_{1}}\right)
$$

where $\boldsymbol{x}^{i_{1}}=\left(x^{i_{1}, 1}, \ldots, x^{i_{1}, r_{i_{1}}}\right)$ are defined over the integral leaves of the eigendistribution $\mathcal{D}_{i_{1}}^{(1)}$ and the remaining ones, namely

$$
\left(\boldsymbol{x}^{1}, \ldots, \boldsymbol{x}^{i_{1}-1}, \boldsymbol{x}^{i_{1}+1}, \ldots, \boldsymbol{x}^{s_{1}}\right)
$$

are coordinates of the leaves, i.e., are constant $\left(\boldsymbol{x}^{1}=\boldsymbol{c}_{1}^{1}, \ldots, \boldsymbol{x}^{i_{1}-1}=\boldsymbol{c}_{1}^{i_{1}-1}, \boldsymbol{x}^{i_{1}+1}=\right.$ $\left.\boldsymbol{c}_{1}^{i_{1}+1}, \ldots, \boldsymbol{x}^{s_{1}}=\boldsymbol{c}_{1}^{s_{1}}\right)$ on each leaf $D_{i_{1}}^{(1)}\left(\boldsymbol{c}_{1}\right)$ of the foliation. Here $\boldsymbol{c}_{1}:=\left(\boldsymbol{c}_{1}^{1}, \ldots, \boldsymbol{c}_{1}^{i_{1}-1}, \boldsymbol{c}_{1}^{i_{1}+1}, \ldots, \boldsymbol{c}_{1}^{s_{1}}\right)$ (the subindex 1 refers to the operator $\boldsymbol{K}_{1}$ ). All operators of the family $\mathcal{K}$ commute; thus, every distribution $\mathcal{D}_{i_{1}}^{(1)}$ is left invariant by the operators $\left\{\boldsymbol{K}_{2}, \ldots, \boldsymbol{K}_{w}\right\}$. This implies that all the operators $\boldsymbol{K}_{\alpha} \in \mathcal{K}$ in the local chart (51) take a block-diagonal form

$$
\left[\boldsymbol{K}_{\alpha}\right]=\left[\begin{array}{l|l|l}
\boldsymbol{K}_{1}^{(\alpha)} & & \\
\hline & \ddots & \\
\hline & & \boldsymbol{K}_{s_{1}}^{(\alpha)}
\end{array}\right], \quad \alpha=1, \ldots, w
$$

where $\left[\boldsymbol{K}_{i_{1}}^{(\alpha)}\right]_{\left.\right|_{D_{i_{1}}^{(1)}\left(c_{1}\right)}}=\left[\boldsymbol{K}_{\left.\alpha\right|_{D_{i_{1}}^{(1)}\left(\boldsymbol{c}_{1}\right)}}\right], i_{1}=1, \ldots, s_{1}$, since the operators $\boldsymbol{K}_{\alpha}$ can be restricted to each leaf $D_{i_{1}}^{(1)}\left(\boldsymbol{c}_{1}\right)$.

Besides, if any other operator of the set $\left\{\boldsymbol{K}_{2}, \ldots, \boldsymbol{K}_{w}\right\}$, say $\boldsymbol{K}_{2}$, has vanishing Haantjes torsion, then the tangent space at any point $\boldsymbol{x}$ admits the finer decomposition

$$
T_{\boldsymbol{x}} M=\bigoplus_{i_{1}, i_{2}}^{s_{1}, s_{2}} \mathcal{D}_{i_{1}}^{(1)}(\boldsymbol{x}) \cap \mathcal{D}_{i_{2}}^{(2)}(\boldsymbol{x})
$$

where $\mathcal{D}_{i_{2}}^{(2)}$ are the generalized eigen-distributions of $\boldsymbol{K}_{2}$, which are integrable by virtue of the Haantjes Theorem 25. Consequently, the Haantjes Theorem can also be applied to the restriction of $\boldsymbol{K}_{2}$ to $D_{i_{1}}^{(1)}\left(\boldsymbol{c}_{1}\right)$. Therefore, there exists a transformation of coordinates, acting only on the coordinates over the leaves of the foliation $D_{i_{1}}^{(1)}$

$$
\Phi: M \rightarrow M, \quad\left(\boldsymbol{x}^{1}, \ldots, \boldsymbol{x}^{i_{1}}, \ldots, \boldsymbol{x}^{s_{1}}\right) \mapsto\left(\boldsymbol{x}^{1}, \ldots, \boldsymbol{y}^{i_{1}}, \ldots, \boldsymbol{x}^{s_{1}}\right)
$$

such that the new coordinates $\boldsymbol{y}^{i_{1}}=\left(y^{i_{1}, 1}, \ldots, y^{i_{1}, r_{i_{1}}}\right)=\boldsymbol{f}^{i_{1}}\left(\boldsymbol{x}^{i_{1}}\right)$ are adapted to the decomposition

$$
T_{\boldsymbol{x}} D_{i_{1}}^{(1)}\left(\boldsymbol{c}_{1}\right)=\bigoplus_{i_{2}}^{s_{2}} \mathcal{D}_{i_{1}}^{(1)}(\boldsymbol{x}) \cap \mathcal{D}_{i_{2}}^{(2)}(\boldsymbol{x}), \quad \boldsymbol{x} \in D_{i_{1}}^{(1)}\left(\boldsymbol{c}_{1}\right)
$$


Thus, we have

$$
\left[\boldsymbol{K}_{i_{1}}^{(\alpha)}\right]=\left[\begin{array}{c|c|c}
\boldsymbol{K}_{i_{1}, 1}^{(\alpha)} & 0 & 0 \\
\hline 0 & \ddots & 0 \\
\hline 0 & 0 & \boldsymbol{K}_{i_{1}, s_{2}}^{(\alpha)}
\end{array}\right], \quad \alpha=1, \ldots, w
$$

where $\left[\boldsymbol{K}_{i_{1}, j}^{(\alpha)}\right]_{D_{D_{1}\left(c_{1}\right) \cap D_{j}^{(2)}\left(c_{2}\right)}^{(1)}}=\left[\boldsymbol{K}_{\left.\alpha\right|_{D_{i_{1}}^{(1)}\left(\boldsymbol{c}_{1}\right) \cap D_{j}^{(2)}\left(\boldsymbol{c}_{2}\right)}}\right], j=1, \ldots, s_{2}$. Let us consider the decomposition

$$
T_{\boldsymbol{x}} M=\bigoplus_{i_{1}, i_{2}}^{s_{1}, s_{2}} \mathcal{D}_{i_{1}}^{(1)}(\boldsymbol{x}) \cap \mathcal{D}_{i_{2}}^{(2)}(\boldsymbol{x})=\bigoplus_{\gamma=1}^{u} \mathcal{U}_{\gamma}(\boldsymbol{x}) \quad \boldsymbol{x} \in M
$$

where in the direct sum (56) $\mathcal{U}_{\gamma} \neq\{\mathbf{0}\}, u \leq n$ and $r_{\gamma}$ denotes the rank of $\mathcal{U}_{\gamma}$ $\left(\sum_{\gamma=1}^{u} r_{\gamma}=n\right)$. Clearly, the distributions $\mathcal{U}_{\gamma}$ are invariant under the action of each operator $\boldsymbol{K}_{\alpha} \in \mathcal{K}$. Besides, these distributions are involutive, and are realized as the intersection of involutive distributions. By assumption, they are also mutually integrable; therefore, there exist local charts in $M$ of the form

$$
\left\{U,\left(y^{1,1}, \ldots, y^{1, r_{1}} ; \ldots ; y^{i_{1}, 1}, \ldots, y^{i_{1}, r_{i_{1}}} ; \ldots ; y^{s_{1}, 1}, \ldots, y^{s_{1}, r_{s_{1}}}\right)\right\}
$$

adapted to the decomposition (56), where all the operators $\boldsymbol{K}_{\alpha} \in \mathcal{K}$ admit simultaneously a (possibly) finer block-diagonal form. By extending the previous procedure to the Haantjes operators $\boldsymbol{K}_{3}, \ldots, \boldsymbol{K}_{w}$, we obtain the decomposition

$$
T_{\boldsymbol{x}} M=\bigoplus_{i_{1}, \ldots, i_{w}}^{s_{1}, \ldots, s_{w}} \mathcal{D}_{i_{1}}^{(1)}(\boldsymbol{x}) \bigcap \ldots \bigcap \mathcal{D}_{i_{w}}^{(w)}(\boldsymbol{x})=\bigoplus_{a=1}^{v} \mathcal{V}_{a}(\boldsymbol{x})
$$

where in the direct sum (58) $\mathcal{V}_{a} \neq\{\mathbf{0}\}, v \leq n$ and $r_{a}$ denotes the rank of $\mathcal{V}_{a}$ $\left(\sum_{a=1}^{v} r_{a}=n\right)$. Then, as the involutive distributions $\mathcal{V}_{a}$ by assumption are mutually integrable, there exist local charts

$$
\left\{U,\left(\boldsymbol{y}^{1}, \ldots, \boldsymbol{y}^{a}, \ldots, \boldsymbol{y}^{v}\right)\right\},
$$

adapted to the decomposition (58), such that

$$
\mathcal{V}_{a}=\left\langle\frac{\partial}{\partial y^{a, 1}}, \ldots, \frac{\partial}{\partial y^{a, r_{a}}}\right\rangle \quad a=1, \ldots, v,
$$

where the natural frame $\left\{\frac{\partial}{\partial y^{a, 1}}, \ldots, \frac{\partial}{\partial y^{a, r_{a}}}\right\}$ over the leaves of $\mathcal{V}_{a}$ is formed by joint generalized eigenvector fields of the operators $\left\{\boldsymbol{K}_{1}, \ldots, \boldsymbol{K}_{w}\right\}$.

Definition 34. Let $\mathcal{K}=\left\{\boldsymbol{K}_{1}, \ldots, \boldsymbol{K}_{w}\right\}$ be a family of Haantjes operators. A Haantjes chart for the family $\mathcal{K}$ is defined to be a local chart $\left\{U,\left(x^{1}, \ldots, x^{n}\right)\right\}$ whose associated natural frame is a generalized eigen-frame of each $\boldsymbol{K}_{\alpha} \in \mathcal{K}$.

The assumption that the generalized eigen-distributions of the Haantjes operators in $\mathcal{K}$ are mutually integrable is sufficient to guarantee the existence of Haantjes charts for the family $\mathcal{K}$. In the next section, we will show that in the case of semisimple Haantjes algebras, such hypothesis is always satisfied.

To conclude this analysis, we prove a result that will be useful for the proof of Theorem 42 of the next section. 
Proposition 35. Let $\boldsymbol{K}_{1}$ and $\boldsymbol{K}_{2}: T M \rightarrow T M$ be two commuting operator fields, and $\mathcal{D}_{i}^{(1)}, \mathcal{D}_{j}^{(2)}, i \in\left\{1, \ldots, s_{1}\right\}, j \in\left\{1, \ldots, s_{2}\right\}$ two involutive generalized eigendistributions of $\boldsymbol{K}_{1}$ and $\boldsymbol{K}_{2}$, with Riesz indices $\rho_{i}$ and $\sigma_{j}$, respectively, which are assumed to be independent of $\boldsymbol{x}$. The following three conditions are equivalent:

(1) the distribution $\mathcal{D}_{i}^{(1)}+\mathcal{D}_{j}^{(2)}$ is involutive;

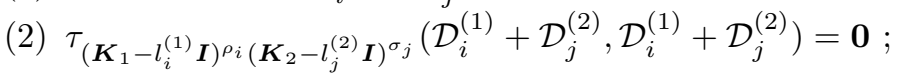

(3) $\mathcal{H}_{\left(\boldsymbol{K}_{1}-l_{i}^{(1)} \boldsymbol{I}\right)^{\rho_{i}}\left(\boldsymbol{K}_{2}-l_{j}^{(2)} \boldsymbol{I}\right)^{\sigma_{j}}}\left(\mathcal{D}_{i}^{(1)}+\mathcal{D}_{j}^{(2)}, \mathcal{D}_{i}^{(1)}+\mathcal{D}_{j}^{(2)}\right)=\mathbf{0}$.

Proof. The proof proceeds as in Theorem 23, with the identification $\boldsymbol{A}:=\left(\boldsymbol{K}_{1}-\right.$ $\left.l_{i}^{(1)} \boldsymbol{I}\right)^{\rho_{i}}, \boldsymbol{B}:=\left(\boldsymbol{K}_{2}-l_{j}^{(2)} \boldsymbol{I}\right)^{\sigma_{j}}$ and exploiting Eqs. (36), (11) and (12).

The latter proposition can be easily extended to an arbitrary number of commuting operators.

\section{HAANTJES ALgEBRAS}

In this section, we shall introduce the notion of Haantjes algebra, which is essentially an associative algebra of operator fields with vanishing Haantjes torsion.

\subsection{Main Definition.}

Definition 36. A Haantjes algebra of rank $m$ is a pair $(M, \mathscr{H})$ with the following properties:

- $M$ is a differentiable manifold of dimension $\mathrm{n}$;

- $\mathscr{H}$ is a set of Haantjes operators $\boldsymbol{K}: T M \rightarrow T M$ that generate

- a free module of rank $\mathrm{m}$ over the ring of smooth functions on $M$ :

$\mathcal{H}_{\left(f \boldsymbol{K}_{1}+g \boldsymbol{K}_{2}\right)}(X, Y)=\mathbf{0}, \quad \forall X, Y \in T M, \quad f, g \in C^{\infty}(M), \quad \forall \boldsymbol{K}_{1}, \boldsymbol{K}_{2} \in \mathscr{H} ;$

- a ring w.r.t. the composition operation

$$
\mathcal{H}_{\left(\boldsymbol{K}_{1} \boldsymbol{K}_{2}\right)}(X, Y)=\mathcal{H}_{\left(\boldsymbol{K}_{2} \boldsymbol{K}_{1}\right)}(X, Y)=\mathbf{0}, \quad \forall \boldsymbol{K}_{1}, \boldsymbol{K}_{2} \in \mathscr{H}, \quad \forall X, Y \in T M .
$$

In addition, if

$$
\boldsymbol{K}_{1} \boldsymbol{K}_{2}=\boldsymbol{K}_{2} \boldsymbol{K}_{1}, \quad \boldsymbol{K}_{1}, \boldsymbol{K}_{2} \in \mathscr{H},
$$

the algebra $(M, \mathscr{H})$ will be said to be an Abelian Haantjes algebra. Moreover, if the identity operator $\boldsymbol{I} \in \mathscr{H}$, then $(M, \mathscr{H})$ will be said to be a Haantjes algebra with identity.

The assumptions (61), (62) ensure that the set $\mathscr{H}$ generates an associative algebra of Haantjes operators. Besides, whenever $\boldsymbol{K} \in \mathscr{H}$, then the powers $\boldsymbol{K}^{i} \in \mathscr{H} \forall i \in \mathbb{N} \backslash\{0\}$.

Let us consider the minimal polynomial of an operator $\boldsymbol{K} \in \mathscr{H}$

$$
m_{\boldsymbol{K}}(\boldsymbol{x}, \lambda)=\prod_{i=1}^{s}\left(\lambda-l_{i}(\boldsymbol{x})\right)^{\rho_{i}}=\lambda^{r}+\sum_{j=1}^{r} c_{j}(\boldsymbol{x}) \lambda^{r-j}, \quad r=\sum_{i=1}^{s} \rho_{i},
$$

where $l_{i}(\boldsymbol{x}), i=1, \ldots, s$ are the pointwise distinct eigenvalues of $\boldsymbol{K}$. The previous observations imply the following results. 
Lemma 37. Let $(M, \mathscr{H})$ be a Haantjes algebra of rank $\mathrm{m}$. If $\boldsymbol{I} \in \mathscr{H}$, then the degree $\mathrm{r}$ of the minimal polynomial of each $\boldsymbol{K} \in \mathscr{H}$ is not greater than $\mathrm{m}$; if $\boldsymbol{I} \notin \mathscr{H}$, then $\mathrm{r} \leq(\mathrm{m}+1)$.

Lemma 38. Let $(M, \mathscr{H})$ be a Haantjes algebra without identity. Then, each $\boldsymbol{K} \in$ $\mathscr{H}$ is a non-invertible operator.

Proof. Let $m_{\boldsymbol{K}}(\boldsymbol{x}, \lambda)$ be the minimal polynomial of $\boldsymbol{K}$, as in Eq. (64). Let us consider the operator $c_{r}(\boldsymbol{x}) \boldsymbol{I}=-\left(\boldsymbol{K}^{r}+\sum_{j=1}^{r-1} c_{j}(\boldsymbol{x}) \boldsymbol{K}^{r-j}\right)$. At the points $\boldsymbol{x} \in M$ where $c_{r}(\boldsymbol{x}) \neq 0$, this operator belongs to $\mathscr{H}$. Then, on this set of points, the identity operator $\boldsymbol{I}$ should also belong to $\mathscr{H}$, which is absurd. Therefore $c_{r}(\boldsymbol{x})=$ $(-1)^{r} \Pi_{i=1}^{s} l_{i}^{\rho_{i}}(\boldsymbol{x})$ vanishes $\forall \boldsymbol{x} \in M$. Consequently, $\boldsymbol{K}$ is not invertible at any point of $M$.

Remark 39. The class of $\omega \mathcal{H}$ manifolds introduced and discussed in [29] can be regarded as a family of symplectic manifolds of dimension $2 n$, endowed with an Abelian Haantjes algebra of rank $m=n$ that fulfills an additional compatibility condition with the symplectic form $\omega$.

The requirements of Definition 36 are apparently demanding and difficult to fulfill in concrete examples. However, a natural Haantjes algebra is realized, in a local chart $\left\{U, \boldsymbol{x}=\left(x^{1}, \ldots, x^{n}\right)\right\}$, by any set of diagonal operators of the form

$$
\boldsymbol{K}=\sum_{k=1}^{n} l_{k}(\boldsymbol{x}) \frac{\partial}{\partial x^{k}} \otimes \mathrm{d} x^{k},
$$

where the smooth functions $l_{k}(\boldsymbol{x})$ play the role of eigenvalue fields of $\boldsymbol{K}$. The operators of the form (65) have vanishing Haantjes torsion and satisfy the differential compatibility condition (61), by virtue of Proposition [12. Moreover, they form a commutative ring, as they also satisfy the relations (62). In fact, such operators generate an algebraic structure that will be said to be a diagonal Haantjes algebra. In the following section, we will show that this interesting structure represents the prototype of Haantjes algebras of commuting semisimple Haantjes operators. This observation suggests the following

Definition 40. A Haantjes algebra $(M, \mathscr{H})$ is said to be semisimple if each operator $\boldsymbol{K} \in \mathscr{H}$ is semisimple.

We prove now a general result concerning the intersections of the kernels of semisimple operator fields, which will be crucial in the proof of Theorem 42 .

Lemma 41. Let $\left\{\boldsymbol{L}_{1}, \ldots, \boldsymbol{L}_{w}\right\}, \boldsymbol{L}_{\alpha}: T M \rightarrow T M, \alpha=1, \ldots, w$ be semisimple (noninvertible) commuting operator fields. Then, there exist $w$ functions $\left(c_{1}, \ldots, c_{w}\right)$ such that

$$
\bigcap_{i=1}^{w} \operatorname{ker} \boldsymbol{L}_{i}=\operatorname{ker}\left(\sum_{i=1}^{w} c_{i} \boldsymbol{L}_{i}\right)
$$

Proof. The operators $\left\{\boldsymbol{L}_{1}, \ldots, \boldsymbol{L}_{w}\right\}$ are semisimple and commute. Therefore there exists a local reference frame where they are, simultaneously, pointwise diagonalizable. Without loss of generality, we can suppose that $\left\{\boldsymbol{L}_{2}, \ldots, \boldsymbol{L}_{s}\right\}$, with $s \leq w$, have kernels whose intersections with $\operatorname{ker} \boldsymbol{L}_{1}$ have strictly decreasing rank. Then, we can choose a local reference frame of common eigenvector fields $\left\{X_{1}, \ldots, X_{n}\right\}$ 
in such a way that the eigenvalue fields of $\left\{\boldsymbol{L}_{1}, \ldots, \boldsymbol{L}_{s}\right\}$, at each $\boldsymbol{x} \in M$ fulfill the conditions

$$
\begin{array}{rlrl}
l_{j}^{(1)}(\boldsymbol{x}) & \neq 0, & j=1, \ldots, r_{1}, \\
l_{j}^{(2)}(\boldsymbol{x}) & \neq 0, \quad j=\left(r_{1}+1\right), \ldots, r_{2}, \\
\ldots & \neq 0 \ldots, \\
l_{j}^{(s)}(\boldsymbol{x}) & \neq 0, \quad j=\left(r_{s-1}+1\right), \ldots, r_{s},
\end{array}
$$

where $r_{1}=\operatorname{rank}\left(\boldsymbol{L}_{1}\right), \ldots, r_{i}=\operatorname{rank}\left(\boldsymbol{L}_{i}\right)_{\mid \operatorname{ker} \boldsymbol{L}_{i-1}}$ for $i=2, \ldots, s$. Then, we can choose $w$ functions $c_{1}, \ldots, c_{w}$ such that $\forall \boldsymbol{x} \in M$ :

$$
\begin{aligned}
c_{j}(\boldsymbol{x})= & 0, \quad j=s+1, \ldots, w, \\
c_{s}(\boldsymbol{x}) \neq & 0, \\
c_{s-1}(\boldsymbol{x}) \neq & -\frac{1}{l_{j}^{(s-1)}(\boldsymbol{x})} c_{s}(\boldsymbol{x}) l_{j}^{(s)}(\boldsymbol{x}), \quad j=\left(r_{s-2}+1\right), \ldots, r_{s-1}, \\
\ldots & \ldots \\
c_{1}(\boldsymbol{x}) \neq & -\frac{1}{l_{j}^{(1)}(\boldsymbol{x})} \sum_{k=2}^{s} c_{k}(\boldsymbol{x}) l_{j}^{(k)}(\boldsymbol{x}), \quad j=1, \ldots, r_{1} .
\end{aligned}
$$

With such choices, the linear combination $\sum_{i=1}^{w} c_{i} \boldsymbol{L}_{i}$ satisfies the property (66).

We are now able to prove a relevant result concerning the integrability of the eigen-distributions of a set of Haantjes operators forming a semisimple, Abelian Haantjes algebra.

Theorem 42. Let $(M, \mathscr{H})$ be a semisimple Abelian Haantjes algebra, and assume that the rank of the eigen-distributions of the operators belonging to $\mathscr{H}$ is independent of $\boldsymbol{x} \in M$. Then, these eigen-distributions, jointly with all of their possible intersections, are mutually integrable.

Proof. Let $\left\{\boldsymbol{K}_{1}, \ldots, \boldsymbol{K}_{m}\right\}$ be a basis of $(M, \mathscr{H})$, and $\left(\mathcal{D}_{i_{1}}^{(1)}, \ldots, \mathcal{D}_{i_{m}}^{(m)}\right), i_{1}=1, \ldots, s_{1}$, $i_{m}=1, \ldots, s_{m}$, be the set of their (proper) eigen-distributions. Let

$$
\mathcal{V}_{a}=\mathcal{D}_{i_{1}}^{(1)}(\boldsymbol{x}) \bigcap \ldots \bigcap \mathcal{D}_{i_{m}}^{(m)}(\boldsymbol{x}) \quad a=1, \ldots, v, \quad v \leq n
$$

denote a nontrivial intersection of eigen-distributions of the operators $\left\{\boldsymbol{K}_{1}, \ldots, \boldsymbol{K}_{m}\right\}$. Consequently, this distribution, being the intersection of distributions which are involutive (due to the Haantjes Theorem), is also involutive. Moreover, since $\mathcal{V}_{a}$ is an intersection of the kernels of the operators $\left(\boldsymbol{K}_{j}-l_{i_{j}}^{(j)} \boldsymbol{I}\right), j=1, \ldots, m$, using Lemma 41 it can be expressed as the kernel of a single Haantjes operator, obtained as a linear combination of $\left\{\boldsymbol{K}_{1}, \ldots, \boldsymbol{K}_{m}\right\}$. Then, the direct sums of the distributions (72) fulfill the condition (3) of Proposition 35] as $(M, \mathscr{H})$ is a Haantjes algebra. Therefore they are mutually integrable.

Theorem 43. Let $\mathcal{K}$ be a family of semisimple commuting operator fields. A set of local coordinates exists such that all the operators of $\mathcal{K}$ can be simultaneously diagonalized if and only if these operators generate an Abelian Haantjes algebra.

Proof. Assume that the operators of $\mathcal{K}$ generate an Abelian Haantjes algebra $(M, \mathscr{H})$. The result follows from Theorem 42 and Proposition 33 by simply observing that the distributions (72), by construction, are formed by proper, joint eigenvector fields of 
a given basis $\left\{\boldsymbol{K}_{1}, \ldots, \boldsymbol{K}_{m}\right\}$ of $\mathscr{H}$. Therefore, the natural frame $\left\{\frac{\partial}{\partial y^{a, 1}}, \ldots, \frac{\partial}{\partial y^{a, r_{a}}}\right\}$ over the leaves of $\mathcal{V}_{a}$, associated to the chart (59), is formed by joint eigenvector fields of the operators $\left\{\boldsymbol{K}_{1}, \ldots, \boldsymbol{K}_{m}\right\}$.

The converse statement can be easily proved by observing that if $\mathcal{K}$ is a family of operators admitting local coordinates in which they all take a diagonal form, they are necessarily Haantjes operators and generate a diagonal Haantjes algebra.

In Section 5.2, we shall present the large class of cyclic Haantjes algebras, which are generated by the powers of a single Haantjes operator. Examples of nondiagonal Haantjes algebras have been studied in 30 .

5.2. Cyclic Haantjes algebras. An especially relevant class of Haantjes algebras is represented by the algebras generated by a single Haantjes operator $\boldsymbol{L}: T M \mapsto$ $T M$. In fact, one can consider the algebra $\mathcal{L}$ of all powers of $\boldsymbol{L}$

$$
\mathcal{L}(\boldsymbol{L}):=\left\langle\boldsymbol{I}, \boldsymbol{L}, \boldsymbol{L}^{2}, \ldots, \boldsymbol{L}^{n-1}, \ldots\right\rangle .
$$

Due to Proposition 16, the algebra $\mathcal{L}(\boldsymbol{L})$ is an example of a Haantjes algebra; we shall call it cyclic Haantjes algebra. Its rank is equal to the degree of the minimal polynomial of $\boldsymbol{L}$, which is not greater than $n$, due to the Cayley-Hamilton theorem.

A natural question is to establish whether a given Haantjes algebra can be generated by a single Haantjes operator. To this aim, we introduce the notion of cyclic generator of $\mathscr{H}$.

Definition 44. Let $(M, \mathscr{H})$ be a rank $\mathrm{m}$ Abelian Haantjes algebra. An operator $\boldsymbol{L}: T M \rightarrow T M$ whose minimal polynomial is of degree $\mathrm{h} \geq \mathrm{m}$ will be said to be a cyclic generator of $\mathscr{H}$ if

$$
\mathscr{H} \subseteq \mathcal{L}(\boldsymbol{L})
$$

Let

$$
\mathcal{B}_{c y c}=\left\{\boldsymbol{I}, \boldsymbol{L}, \boldsymbol{L}^{2}, \ldots, \boldsymbol{L}^{h-1}\right\},
$$

be a cyclic basis of $\mathcal{L}(\boldsymbol{L})$. A basis $\mathcal{B}$ of $\mathscr{H}$ such that $\mathcal{B} \subseteq \mathcal{B}_{\text {cyc }}$ will be said to be a cyclic basis of $\mathscr{H}$.

A cyclic basis allows us to represent any Haantjes operator $\boldsymbol{K} \in \mathscr{H}$ as a polynomial field in $\boldsymbol{L}$ of degree at most $(h-1)$, i.e.

$$
\boldsymbol{K}=p_{\boldsymbol{K}}(\boldsymbol{x}, \boldsymbol{L})=\sum_{k=0}^{h-1} a_{k}(\boldsymbol{x}) \boldsymbol{L}^{k},
$$

where $a_{k}(\boldsymbol{x}) \in C^{\infty}(M)$. We propose now a characterization of semisimple operators, with real eigenvalues, belonging to a cyclic algebra.

Proposition 45. Let $\boldsymbol{L}: T M \rightarrow T M$ be a semisimple operator with $h$ pointwise distinct eigenvalues $\left\{\lambda_{1}(\boldsymbol{x}), \ldots, \lambda_{h}(\boldsymbol{x})\right\}$. Let $\boldsymbol{K}: T M \rightarrow T M$ be another operator field possessing $s$ pointwise distinct eigenvalues, with $s \leq h$. The following conditions are equivalent:

- $\boldsymbol{K}$ belongs to the cyclic algebra of rank $\mathrm{h}$ generated by $\boldsymbol{L}$, i.e.

$$
\boldsymbol{K} \in \mathcal{L}(\boldsymbol{L}) ;
$$

- there exists a polynomial field $p_{\boldsymbol{K}}(\boldsymbol{x}, \lambda)$ in $\lambda$ of degree at most $(h-1)$, such that

$$
\boldsymbol{K}=p_{\boldsymbol{K}}(\boldsymbol{x}, \boldsymbol{L})
$$


- each eigen-distribution of $\boldsymbol{L}$ is included in a single eigen-distribution of $\boldsymbol{K}$ :

$$
\mathcal{C}_{\lambda_{i}}:=\operatorname{ker}\left(\boldsymbol{L}-\lambda_{i} \boldsymbol{I}\right) \subseteq \mathcal{D}_{l_{i}}:=\operatorname{ker}\left(\boldsymbol{K}-l_{i} \boldsymbol{I}\right),
$$

where it is understood that the eigenvalues $\left(l_{1}(\boldsymbol{x}), \ldots, l_{h}(\boldsymbol{x})\right)$ of $\boldsymbol{K}$ may not be all distinct.

Proof. The equivalence between conditions (75) and (76) holds since the minimal polynomial of $\boldsymbol{L}$ is given by

$$
m_{\boldsymbol{L}}(\boldsymbol{x}, \lambda)=\Pi_{i=1}^{h}\left(\lambda-\lambda_{i}(\boldsymbol{x})\right)
$$

and the set (73) is a basis of $\mathcal{L}(\boldsymbol{L})$. Besides, condition (76) implies (77) as every eigenvector field $X$ of $\boldsymbol{L}$ belonging to $\mathcal{C}_{\lambda_{i}}$ is also an eigenvector field of $\boldsymbol{K}$, with eigenvalue $l_{i}(\boldsymbol{x})=p_{\boldsymbol{K}}\left(\boldsymbol{x}, \lambda_{i}\right)$ :

$$
\boldsymbol{K} X=p_{\boldsymbol{K}}(\boldsymbol{x}, \boldsymbol{L}) X=p_{\boldsymbol{K}}\left(\boldsymbol{x}, \lambda_{i}\right) X .
$$

Vice versa, assuming that condition (77) is satisfied, it suffices to show that there exists a polynomial $p_{\boldsymbol{K}}(\boldsymbol{x}, \lambda), \lambda \in \mathbb{R}$, such that $\boldsymbol{K}$ and $p_{\boldsymbol{K}}(\boldsymbol{x}, \boldsymbol{L})$ agree on a basis adapted to the decomposition

$$
T_{\boldsymbol{x}} M=\bigoplus_{i=1}^{h} \mathcal{C}_{\lambda_{i}}(\boldsymbol{x})
$$

To this aim, we must solve the following system

$$
l_{i}(\boldsymbol{x})=p_{\boldsymbol{K}}\left(\boldsymbol{x}, \lambda_{i}\right)=\sum_{k=0}^{h-1} a_{k}(\boldsymbol{x}) \lambda_{i}^{k}(\boldsymbol{x}), \quad i=1, \ldots, h .
$$

This can be done by means of the $h$ Lagrange interpolation polynomials of degree $(h-1)$

$$
\pi_{i}(\lambda)=\frac{\Pi_{j \neq i}^{h}\left(\lambda-\lambda_{j}\right)}{\Pi_{j \neq i}^{h}\left(\lambda_{i}-\lambda_{j}\right)}, \quad i=1, \ldots, h
$$

which yield the expressions

$$
p_{\boldsymbol{K}}(\boldsymbol{x}, \lambda)=\sum_{i=1}^{h} l_{i}(\boldsymbol{x}) \pi_{i}(\lambda) .
$$

Therefore, we obtain the relation

$$
\boldsymbol{K}=\sum_{i=1}^{h} p_{\boldsymbol{K}}\left(\boldsymbol{x}, \lambda_{i}\right) \pi_{i}(\boldsymbol{L})
$$

where $\pi_{i}(\boldsymbol{L})$ are pointwise projection operator fields onto the distribution $\mathcal{C}_{\lambda_{i}}$. The basis $\mathcal{B}_{\text {int }}=\left\{\pi_{1}(\boldsymbol{L}), \ldots, \pi_{h}(\boldsymbol{L})\right\}$ will be said to be a Lagrange interpolation basis of $\mathcal{L}(\boldsymbol{L})$.

Corollary 46. If one of the equivalent conditions of Proposition 45 is satisfied and $\boldsymbol{L}$ is a Haantjes operator, then the operator $\boldsymbol{K}$ is also a Haantjes operator which commutes with $\boldsymbol{L}$. In addition, every integrable eigen-frame of $\boldsymbol{L}$ is also an integrable eigen-frame for $\boldsymbol{K}$. Therefore we have

$$
\boldsymbol{L}_{\left.\right|_{C_{j}(\boldsymbol{x})}}=\lambda_{j} \boldsymbol{I}_{\left.\right|_{C_{j}(\boldsymbol{x})}}, \quad \boldsymbol{K}_{\left.\right|_{C_{j}(\boldsymbol{x})}}=p_{\boldsymbol{K}}\left(\boldsymbol{x}, \lambda_{j}\right) \boldsymbol{I}_{\left.\right|_{C_{j}(\boldsymbol{x})}},
$$

where $C_{j}(\boldsymbol{x})$ denotes an integral leaf, passing through $\boldsymbol{x}$, of the eigen-distribution $\mathcal{C}_{\lambda_{j}}$ of $\boldsymbol{L}$ 
Thus, given a semisimple Haantjes operator $\boldsymbol{K}$ with $s$ pointwise distinct eigenvalues $\left\{l_{1}(\boldsymbol{x}), \ldots, l_{s}(\boldsymbol{x})\right\}$, one can always construct another Haantjes operator $\boldsymbol{L}$ fulfilling the condition (76), by considering a finer (or at least no coarser) decomposition than the spectral decomposition of $\boldsymbol{K}$

$$
T_{\boldsymbol{x}} M=\bigoplus_{i=1}^{s} \mathcal{D}_{l_{i}}(\boldsymbol{x})
$$

according to which

$$
\boldsymbol{K}=\sum_{i=1}^{s} l_{i} \boldsymbol{P}_{i}(\boldsymbol{K})
$$

Here $\boldsymbol{P}_{i}$ are pointwise projection operators onto $\mathcal{D}_{l_{i}}$. To this aim, let us consider a local chart $\left\{U,\left(\boldsymbol{x}^{1}, \ldots, \boldsymbol{x}^{s}\right)\right\}$, adapted to the characteristic web of $\boldsymbol{K}$; thus, it fulfills Eq. (45). By way of an example, one can consider the further decomposition

$$
\mathcal{D}_{l_{i}}(\boldsymbol{x})=\bigoplus_{j_{i}=1}^{r_{i}} \mathcal{C}_{i, j_{i}}(\boldsymbol{x}), \quad r_{i}=\operatorname{rank} \mathcal{D}_{l_{i}}
$$

in terms of one-dimensional Lie subalgebras

$$
\mathcal{C}_{i, j_{i}}=\left\langle\frac{\partial}{\partial x^{i, j_{i}}}\right\rangle \subseteq \mathcal{D}_{l_{i}}
$$

Then, one can construct the operator

$$
\boldsymbol{L}=\sum_{i=1}^{s} \lambda_{i, j_{i}} \sum_{j_{i}=1}^{r_{i}} \frac{\partial}{\partial x^{i, j_{i}}}=\sum_{i=1, j_{i}=1}^{s, r_{i}} \lambda_{i, j_{i}} \boldsymbol{\pi}_{i, j_{i}}
$$

where $\boldsymbol{\pi}_{i, j_{i}}$ are projection operators onto the subalgebras $\mathcal{C}_{i, j_{i}}$, and $\lambda_{i, j_{i}}$ are arbitrarily chosen (but pointwise distinct) functions, numbered according to the finer decomposition (81) of $T_{\boldsymbol{x}} M$. These functions will play the role of eigenvalues of $\boldsymbol{L}$. Consequently, we have

$$
\boldsymbol{P}_{i}=\sum_{j_{i}=1}^{r_{i}} \boldsymbol{\pi}_{i, j_{i}}
$$

Thus, $p\left(\lambda_{i, j_{i}}\right)=l_{i}, i=1, \ldots, s, j_{i}=1, \ldots, r_{i}$.

Finally, as a consequence of Proposition 45, we have the following result.

Proposition 47. Every semisimple Abelian Haantjes algebra $(M, \mathcal{H})$ is cyclic and admits a cyclic Nijenhuis generator.

Proof. Let us consider the Haantjes chart

$$
\left\{U, \boldsymbol{x}=\left(\boldsymbol{y}^{1}, \ldots, \boldsymbol{y}^{v}\right)\right\},
$$

adapted to the spectral decomposition

$$
T_{\boldsymbol{x}} M=\bigoplus_{a=1}^{v} \mathcal{V}_{a}(\boldsymbol{x})
$$

whose existence is guaranteed by Theorem 43. Let us denote by $m$ the rank of $(M, \mathcal{H})$. If $v \geq m$, each operator of the form

$$
\boldsymbol{L}=\sum_{a=1}^{v} \lambda_{a}(\boldsymbol{x}) \sum_{j_{a}=1}^{r_{a}} \frac{\partial}{\partial y^{a, j_{a}}} \otimes \mathrm{d} y^{a, j_{a}}
$$


is a cyclic Haantjes generator of $\mathscr{H}$, provided that its $v$ eigenvalue fields $\left\{\lambda_{1}(\boldsymbol{x}), \ldots, \lambda_{v}(\boldsymbol{x})\right\}$ are arbitrary but distinct smooth functions at any point of $U$. In fact, the eigendistributions of the operator (84) are the distributions $\mathcal{V}_{a}$; consequently, by construction they satisfy condition (77). Moreover, this operator also satisfies the assumptions of Proposition 45, In particular, if the eigenvalues of $\boldsymbol{L}$ are chosen to be

$$
\lambda_{a}(\boldsymbol{x})=\lambda_{a}\left(y^{a, 1}, \ldots, y^{a, r_{a}}\right) \quad a=1, \ldots, v,
$$

then $\boldsymbol{L}$ is a cyclic Nijenhuis generator, that is, its Nijenhuis torsion identically vanishes, due to Lemma 9. If $v<m$, a cyclic generator can still be constructed, because we can further decompose some of the distributions $\mathcal{V}_{a}$, as in Eq. (60), into a direct sum of mutually integrable sub-distributions. Precisely, we have

$$
\mathcal{V}_{a}=\left\langle\frac{\partial}{\partial y^{a, 1}}, \ldots, \frac{\partial}{\partial y^{a, r_{a}}}\right\rangle=\bigoplus_{i_{a}=1}^{\bar{r}_{i_{a}}}\left\langle\frac{\partial}{\partial y^{a, 1}}, \ldots, \frac{\partial}{\partial y^{a, i_{a}}}\right\rangle=\bigoplus_{i_{a}=1}^{\bar{r}_{i_{a}}} \mathcal{C}_{a, i_{a}}
$$

with $\sum \bar{r}_{j_{a}}=r_{a}$, and the decomposition of $\mathcal{V}_{a}$ can be realized in such a way that the number of addends appearing into the direct sum

$$
T_{\boldsymbol{x}} M=\bigoplus_{a=1, i_{a}=1}^{v, \bar{r}_{i_{a}}} \mathcal{C}_{a, i_{a}}
$$

again equals $m$.

\section{HaAntjes algebra for the Coulomb-Kepler potential}

In this section, we shall illustrate the novel example of the Haantjes algebra associated with a general class of integrable systems. As a particular instance of this class, we shall discuss a fundamental physical model: the Coulomb-Kepler system in the configuration space given by the affine $3 \mathrm{D}$ space $\mathcal{A}_{3}$. The most general Hamiltonian function separable in spherical coordinates $\left\{\left(q_{1}=r, q_{2}=\theta, q_{3}=\phi\right)\right\}$ reads

$$
H=\frac{1}{2 m}\left(p_{r}+\frac{p_{\theta}^{2}}{r^{2}}+\frac{p_{\phi}^{2}}{r^{2} \sin ^{2} \theta}\right)+e(r)+\frac{h(\theta)}{r^{2}}+\frac{s(\phi)}{r^{2} \sin ^{2} \theta},
$$

where $e(r), h(\theta), s(\phi)$ are arbitrary smooth functions of their argument. Let us consider the semisimple Haantjes algebra of rank three over the phase space $T^{*} \mathcal{A}_{3}$, generated by the identity operator $\boldsymbol{I}$, and

$$
\begin{aligned}
& \boldsymbol{K}_{1}=r^{2} \sin ^{2} \theta\left(\frac{\partial}{\partial \phi} \otimes \mathrm{d} \phi+\frac{\partial}{\partial p_{\phi}} \otimes \mathrm{d} p_{\phi}\right), \\
& \boldsymbol{K}_{2}=r^{2}\left(\frac{\partial}{\partial \theta} \otimes \mathrm{d} \theta+\frac{\partial}{\partial p_{\theta}} \otimes \mathrm{d} p_{\theta}+\frac{\partial}{\partial \phi} \otimes \mathrm{d} \phi+\frac{\partial}{\partial p_{\phi}} \otimes \mathrm{d} p_{\phi}\right) .
\end{aligned}
$$

This Haantjes algebra allows us to construct a $\omega \mathscr{H}$ algebra, in the spirit of Ref. [29], and in particular the Lenard-Haantjes chain of length three formed by

$$
\boldsymbol{I}^{T} \mathrm{~d} H=\mathrm{d} H, \quad \boldsymbol{K}_{1}^{T} \mathrm{~d} H=\mathrm{d} I_{1}, \quad \boldsymbol{K}_{2}^{T} \mathrm{~d} H=\mathrm{d} I_{2} .
$$

It represents a chain of exact one-forms whose potential functions are the independent integrals of motion in involution

$$
H, \quad I_{1}=\frac{p_{\phi}^{2}}{2 m}+s(\phi), \quad I_{2}=\frac{1}{2 m}\left(p_{\theta}^{2}+\frac{p_{\phi}^{2}+s(\phi)}{\sin ^{2} \theta}\right)+h(\theta)
$$


for the Hamiltonian system with Hamiltonian function (87).

If $e(r)=-k / r$ (for a suitable constant $k$ ), $h(\theta)=s(\phi)=0$, one gets the classical Coulomb-Kepler system. For such model, the authors of [24] proved that a Nijenhuis recursion operator compatible with the original symplectic structure can not exist. Indeed, the recursion operator they found provides us with a Lenard chain of integrals of motion which are not independent of the Hamiltonian (87).

\section{Cyclic Haantues operators: Example}

We shall present here an example illustrating some interesting aspects of the Haantjes geometry previously discussed. In particular, as an application of the main theorems, we develop the procedure of constructing a Haantjes chart and a cyclic generator for a semisimple Haantjes algebra of rank 2, which has been exploited in the study of the Jacobi-Calogero Hamiltonian system [32].

7.1. Construction of a Haantjes chart. Let us consider the affine $3 \mathrm{D}$ space $\mathcal{A}_{3}$, with a cartesian coordinate system $\{O ; \boldsymbol{x}=(x, y, z)\}$ and the two operators $\boldsymbol{K}_{i}: T M \rightarrow T M$ defined as

$$
\begin{gathered}
\boldsymbol{K}_{1}:=y^{2} \frac{\partial}{\partial x} \otimes d x+x^{2} \frac{\partial}{\partial y} \otimes d y-x y\left(\frac{\partial}{\partial x} \otimes d y+\frac{\partial}{\partial y} \otimes d x\right), \\
\boldsymbol{K}_{2}:=\left(y^{2}+z^{2}\right) \frac{\partial}{\partial x} \otimes d x+\left(x^{2}+z^{2}\right) \frac{\partial}{\partial y} \otimes d y+\left(x^{2}+y^{2}\right) \frac{\partial}{\partial z} \otimes d z \\
-x y\left(\frac{\partial}{\partial x} \otimes d y+\frac{\partial}{\partial y} \otimes d x\right)-x z\left(\frac{\partial}{\partial x} \otimes d z+\frac{\partial}{\partial z} \otimes d x\right)-y z\left(\frac{\partial}{\partial y} \otimes d z+\frac{\partial}{\partial z} \otimes d y\right) .
\end{gathered}
$$

They generate an Abelian Haantjes algebra $\left(\mathcal{A}_{3}, \mathscr{H}\right)$ of rank 2 , as

$$
\boldsymbol{K}_{1}^{2}=f \boldsymbol{K}_{1}, \quad \boldsymbol{K}_{2}^{2}=g \boldsymbol{K}_{2}, \quad \boldsymbol{K}_{1} \boldsymbol{K}_{2}=g \boldsymbol{K}_{1},
$$

where

$$
f(\boldsymbol{x})=x^{2}+y^{2}, \quad g(\boldsymbol{x})=x^{2}+y^{2}+z^{2} .
$$

Note that $\boldsymbol{K}_{2}$ is a special case of the inertia tensor (27), for of a single mass point $P_{\gamma} \equiv O$ with unitary mass, and $n=3$. Interestingly enough, the two operators $\boldsymbol{K}_{1}$ and $\boldsymbol{K}_{2}$ are the projections onto the space $\mathcal{A}_{3}$ of the operators (88) and (89), defined over $T^{*} \mathcal{A}_{3}$, which are associated with the model (87). The spectra of $\boldsymbol{K}_{1}$ and $\boldsymbol{K}_{2}$ are

$$
\begin{array}{lr}
\operatorname{Spec}\left(\boldsymbol{K}_{1}\right)= & \left\{l_{1}^{(1)}=f, l_{2}^{(1)}=0\right\} \\
\operatorname{Spec}\left(\boldsymbol{K}_{2}\right)= & \left\{l_{1}^{(2)}=0, l_{2}^{(2)}=g\right\},
\end{array}
$$

and their eigen-distributions read

$$
\begin{gathered}
\mathcal{D}_{1}^{(1)}=\left\langle Y_{1}\right\rangle, \quad \mathcal{D}_{2}^{(1)}=\left\langle Y_{2}, Y_{3}\right\rangle, \quad Y_{1}:=-y \frac{\partial}{\partial x}+x \frac{\partial}{\partial y}, Y_{2}:=x \frac{\partial}{\partial x}+y \frac{\partial}{\partial y}, Y_{3}:=\frac{\partial}{\partial z}, \\
\mathcal{D}_{1}^{(2)}=\left\langle Z_{1}\right\rangle, \quad \mathcal{D}_{2}^{(2)}=\left\langle Z_{2}, Z_{3}\right\rangle, \quad Z_{1}:=Y_{2}+z Y_{3}, Z_{2}:=Y_{1}, Z_{3}:=-z \frac{\partial}{\partial x}+x \frac{\partial}{\partial z} .
\end{gathered}
$$


We can construct Haantjes charts for the operators $\boldsymbol{K}_{1}$ and $\boldsymbol{K}_{2}$ by determining coordinates adapted to the decomposition (49). To this aim, we observe that

$$
\begin{gathered}
\mathcal{D}_{1}^{(1)} \cap \mathcal{D}_{1}^{(2)}=\langle 0\rangle, \quad \mathcal{D}_{1}^{(1)} \cap \mathcal{D}_{2}^{(2)}=\left\langle Z_{2}\right\rangle, \quad \mathcal{D}_{2}^{(1)} \cap \mathcal{D}_{1}^{(2)}=\left\langle Z_{1}\right\rangle, \\
\mathcal{D}_{2}^{(1)} \cap \mathcal{D}_{2}^{(2)}=\left\langle Z_{4}\right\rangle, \quad Z_{4}=-x z \frac{\partial}{\partial x}-y z \frac{\partial}{\partial y}+\left(x^{2}+y^{2}\right) \frac{\partial}{\partial z} .
\end{gathered}
$$

Therefore, the tangent spaces can be decomposed as

$$
T_{\boldsymbol{x}} M=\left\langle Z_{1}\right\rangle(\boldsymbol{x}) \oplus\left\langle Z_{2}\right\rangle(\boldsymbol{x}) \oplus\left\langle Z_{4}\right\rangle(\boldsymbol{x}) .
$$

A set of local coordinates adapted to such decomposition is given (for $x \neq 0$ ) by the functions

$$
\begin{aligned}
& x_{1}=\frac{y}{x} \quad \Rightarrow \mathrm{d} x_{1} \in\left(\left\langle Z_{1}\right\rangle \oplus\left\langle Z_{4}\right\rangle\right)^{\circ} \\
& x_{2}=\sqrt{x^{2}+y^{2}+z^{2}} \quad \Rightarrow \mathrm{d} x_{2} \in\left(\left\langle Z_{2}\right\rangle \oplus\left\langle Z_{4}\right\rangle\right)^{\circ} \\
& x_{3}=\frac{z}{\sqrt{x^{2}+y^{2}+z^{2}}} \quad \Rightarrow \mathrm{d} x_{3} \in\left(\left\langle Z_{1}\right\rangle \oplus\left\langle Z_{2}\right\rangle\right)^{\circ} .
\end{aligned}
$$

Such coordinates are related to spherical coordinates $(r, \theta, \phi)$ in $\mathcal{A}_{3}$ as

$$
x_{1}=\tan \phi, \quad x_{2}=r, \quad x_{3}=\cos \theta .
$$

They are characteristic functions of the spherical web whose fibers are the three foliations generated by:

- $\left\langle Z_{1}\right\rangle \oplus\left\langle Z_{4}\right\rangle$, half-planes issued from the $z$ axis;

- $\left\langle Z_{2}\right\rangle \oplus\left\langle Z_{4}\right\rangle$, spheres centered in $O$;

- $\left\langle Z_{1}\right\rangle \oplus\left\langle Z_{2}\right\rangle$ one-folded circular cones with axis $z$ and vertex $O$.

In these coordinates, the Haantjes operators $\boldsymbol{K}_{1}$ and $\boldsymbol{K}_{2}$ take the diagonal form

$$
\begin{array}{r}
\boldsymbol{K}_{1}=x_{2}^{2}\left(1-x_{3}^{2}\right) \frac{\partial}{\partial x_{1}} \otimes d x_{1} \\
\boldsymbol{K}_{2}=x_{2}^{2}\left(\frac{\partial}{\partial x_{1}} \otimes d x_{1}+\frac{\partial}{\partial x_{3}} \otimes d x_{3}\right) .
\end{array}
$$

A cyclic Haantjes generator (admitting three distinct eigenvalues) for the Haantjes algebra $\mathscr{H}$ is given by

$$
\boldsymbol{L}=\boldsymbol{K}_{1}+\boldsymbol{K}_{2}
$$

whose spectrum is

$$
\operatorname{Spec}(\boldsymbol{L})=\left\{\lambda_{1}=-x_{2}^{2}\left(x_{3}^{2}-2\right), \lambda_{2}=0, \lambda_{3}=x_{2}^{2}\right\} .
$$

The corresponding eigen-distributions are $\left\langle Z_{2}\right\rangle,\left\langle Z_{1}\right\rangle,\left\langle Z_{4}\right\rangle$, respectively. In fact

$$
\boldsymbol{K}_{1}=\left(1+\frac{\lambda_{3}}{\lambda_{1}}\right) \boldsymbol{L}-\frac{1}{\lambda_{1}} \boldsymbol{L}^{2},
$$

and

$$
\boldsymbol{K}_{2}=-\frac{\lambda_{3}}{\lambda_{1}} \boldsymbol{L}+\frac{1}{\lambda_{1}} \boldsymbol{L}^{2} .
$$

Thus, $\mathscr{H}$ turns out to be a Haantjes subalgebra of the cyclic Haantjes algebra of rank three

$$
\mathcal{L}(\boldsymbol{L})=\left\langle\boldsymbol{I}, \boldsymbol{L}, \boldsymbol{L}^{2}\right\rangle=\left\langle\boldsymbol{I}, \boldsymbol{K}_{1}, \boldsymbol{K}_{2}\right\rangle .
$$


The eigenvalues of $\boldsymbol{L}$ are such that $\boldsymbol{L}$, in the original cartesian coordinates $(x, y, z)$, takes a simple polynomial form. Precisely, we have

$$
\begin{aligned}
\boldsymbol{L}= & \left(2 y^{2}+z^{2}\right) \frac{\partial}{\partial x} \otimes d x+\left(2 x^{2}+z^{2}\right) \frac{\partial}{\partial y} \otimes d y+\left(x^{2}+y^{2}\right) \frac{\partial}{\partial z} \otimes d z \\
& -2 x y\left(\frac{\partial}{\partial x} \otimes d y+\frac{\partial}{\partial y} \otimes d x\right)-x z\left(\frac{\partial}{\partial x} \otimes d z+\frac{\partial}{\partial z} \otimes d x\right) \\
& -y z\left(\frac{\partial}{\partial y} \otimes d z+\frac{\partial}{\partial z} \otimes d y\right) .
\end{aligned}
$$

The cyclic Haantjes algebra with basis (93) also admits cyclic Nijenhuis generators, as for instance the operator

$$
\boldsymbol{N}=x_{1} \frac{\partial}{\partial x_{1}} \otimes d x_{1}+x_{2}^{2} \frac{\partial}{\partial x_{2}} \otimes d x_{2}+x_{3}^{2} \frac{\partial}{\partial x_{3}} \otimes d x_{3} .
$$

However, although it takes a rational form in the original cartesian coordinates $\{x, y, z\}$, the expression of this operator turns out to be much more complicated than that of $\boldsymbol{L}$. In fact, the numerators of its components are polynomials up to degree 9. This shows that exploiting Haantjes (with non-vanishing Nijenhuis torsion) cyclic generators can be computationally more convenient than using Nijenhuis cyclic generators.

\section{A COMPARISON With Other ALgebraic StruCtures: HaAntJes MANIFOLDS AND KILLING-STÄCKEL ALGEBRAS}

It could be useful to compare our definition of Haantjes algebras over a differentiable manifold, proposed in Section 5, with the notion of Haantjes manifolds recently introduced by Magri 20]. The main difference between the two constructions resides in their distinct degree of generality, which obviously reflects the different motivations underlying the two approaches.

In our construction of Haantjes algebras, we are mainly concerned with the abstract, general theory of commuting Haantjes operators, without any reference to additional geometric structures like exact 1-forms or symmetry vector fields, that in Magri's theory are essential in order to define Lenard complexes of commuting vector fields or exact 1-forms [21, 22.

In other words, although Magri's Haantjes manifolds possess a richer axiomatic structure than our Haantjes algebras, we prefer to articulate our definition on a minimal number of requirements, in order to have a flexible structure, which in a subsequent step can be made suitable for the study of more specific problems, as the separation of variables in the context of integrability or Riemannian geometry. Keeping with this philosophy, we postpone the introduction of additional geometric structures (as Haantjes chains of exact 1-forms, symplectic forms 29], Poisson bivectors [31] or Riemannian metrics [32]) to a further stage of the theory.

Another relevant aspect is that the notion of Killing-Stäckel algebra on an ndimensional Riemannian manifold, due to Benenti et al. [3], can be naturally interpreted in terms of Haantjes algebras of rank $n$. In order to compare the two notions, it is useful to observe that the cyclic generator (92) enjoys a special property: In fact, its contravariant form is a Killing two-tensor with respect to the Euclidean metric of the affine space $\mathcal{A}_{3}$. So, we shall call it a Killing-Haantjes

cyclic generator; it can be identified with a characteristic tensor (CKT) of the 
Killing-Stäckel algebra (93). Thus, the theory of Haantjes cyclic algebras over a Riemannian manifold makes contact with the notion of Killing-Stäckel algebras, which offer a geometrical setting for the classical theory of separation of variables for Hamiltonian systems going back to Eisenhart, Stäckel, Jacobi, etc. The main difference between the two algebraic structures is that Killing-Stäckel algebras are vector spaces of Killing two-tensors over $\mathbb{R}$. Instead, Haantjes algebras are modules over $C^{\infty}(M)$ of Haantjes operators.

At the same time, it is interesting to notice that, starting from a KillingHaantjes cyclic generator, one can choose suitable functions to generate other Killing-Haantjes two-tensors, that is, elements of Killing-Stäckel algebras. The conditions which such functions must obey are under investigation.

To summarize the comparison among these geometric structures, we can distinguish three different scenarios.

(i) When dealing with Killing-Stäckel algebras, we are realizing a specific Haantjes algebra with constant coefficients, which does not always possess a cyclic KillingHaantjes generator. In fact, although a CKT does exist, it is not always a cyclic generator, since to reconstruct the full algebra one would need to combine the powers of the CKT with suitable functions. Instead, in Killing-Stäckel algebras only linear combinations with constant coefficients are allowed (by definition).

(ii) One can consider a generalization of the Killing-Stäckel algebra, obtained combining the powers of the CKT by means of functions. In this case one obtains a larger algebra, which is a full cyclic Haantjes algebra, defined over the ring of smooth functions.

(iii) The most general case is that of Haantjes algebras that do not come from Killing tensors.

\section{ACKNOWLEDGMENTS}

The authors wish to thank F. Magri for having drawn our attention to the theory of Haantjes manifolds and N. Kamran for discussions, a reading of the manuscript and encouragement.

We also thank heartily Y. Kosmann-Schwarzbach for letting us know the preprint 18 before its publication.

The research of P. T. has been supported by the research project PGC2018094898-B-I00, Ministerio de Ciencia, Innovación y Universidades, Spain, and by the ICMAT Severo Ochoa project SEV-2015-0554, Ministerio de Ciencia, Innovación y Universidades, Spain. P. T. and G. T. are members of Gruppo Nazionale di Fisica Matematica (GNFM) of INDAM.

\section{REFERENCES}

[1] S. Benenti, Inertia tensors and Stackel systems in the Euclidean spaces, Rend. Sem. Mat. Univ. Politec. Torino 50, 315-341 (1992).

[2] S. Benenti, Orthogonal separable dynamical Systems, Math. Publ., Silesian Univ. Opava 1 , 163-184 (1993).

[3] S. Benenti, C. Chanu, and G. Rastelli, Remarks on the connection between the additive separation of the Hamilton-Jacobi equation and the multiplicative separation of the Schröedinger equation. I. The completeness and Robertson conditions, J. Math. Phys. 43, 5183-5222 (2002).

[4] O.I. Bogoyavlenskij, Necessary Conditions for Existence of Non-Degenerate Hamiltonian Structures, Commun. Math. Phys 182, 253-290 (1996). 
[5] O.I. Bogoyavlenskij, General algebraic identities for the Nijenhuis and Haantjes torsions, Izvestya Mathematics 68, 1129-1141 (2004).

[6] O.I. Bogoyavlenskij, Schouten tensor and bi-Hamiltonian systems of hydrodynamic type, J. Math. Phys 47, paper n. 023504, 14 pages (2006).

[7] O.I. Bogoyavlenskij, Block-diagonalizability problem for hydrodynamic type systems, J. Math. Phys 47, paper n. 063502, 9 pages (2006).

[8] A.V. Bolsinov, A.Y. Konyaev and V.S. Matveev, Nijenhuis Geometry, Preprint arXiv: 1903.04603 (2019).

[9] M. De Filippo, G. Vilasi, and M. Salerno, A geometrical approach to the integrability of soliton equations, Lett. Math. Phys. 9, 85-91 (1985).

[10] G. Falqui, M. Pedroni, Separation of variables for bi-hamiltonian systems, Math. Phys. Anal. Geom. 6, 139-179 (2003).

[11] E.V. Ferapontov and D.G. Marshall, Differential-geometric approach to the integrability of hydrodynamics chains: the Haantjes tensor, Mat. Ann. 339, 61-99 (2007).

[12] A. Frolicher and A. Nijenhuis, Theory of Vector-Valued Differential Forms. Part I, Indag. Mathematicae 18, 338-359 (1956).

[13] V.S. Gerdjikov, G. Vilasi and A.B. Yanovski, Integrable Hamiltonian Hierarchies, Lect. Not. Phys., Vol. 748 Berlin Heidelberg: Springer, 2008.

[14] M. Gorgone, F. Oliveri, M.P. Speciale, On the decoupling problem of general quasilinear first order systems in two independent variables, J. Math. Anal. Appl. 446, 276-298 (2017).

[15] J. Haantjes, On $X_{n-1}$-forming sets of eigenvectors, Indag. Mathematicae 17, 158-162 (1955).

[16] A. Ibort, F. Magri, G. Marmo, Bi-hamiltonian structures and Stäckel separability, J. Geom. Phys. 33, 210-228 (2000).

[17] N. Jacobson, Basic Algebra 2 (2nd ed.), Dover (2009).

[18] Y. Kosmann-Schwarzbach, Beyond recursion operators, Preprint arXiv:1712.08908, 2017, in Trends in Mathematics, Geometric Methods in Physics XXXVI, Workshop and Summer School, Białowieża, Poland, July 2017, Eds. P. Kielonowski, A. Odzijewicz, E. Previato, Birkhauser, Basel (2019).

[19] F. Magri, Recursion operators and Frobenius manifolds, SIGMA 8, paper 076, 7 pages (2012).

[20] F. Magri, Haantjes manifolds, Journal of Physics: Conference Series 482, paper 012028, 10 pages (2014)

[21] F. Magri, WDVV Equations, Il Nuovo Cimento 38 C, paper 166, 10 pages (2015).

[22] F. Magri, Haantjes manifolds and Veselov Systems, Theor. Math. Phys. 189, 1486-1499 (2016).

[23] F. Magri, Haantjes manifolds with Symmetry, Theor. Math. Phys. 196, 1217-1229 (2018).

[24] G. Marmo and G. Vilasi, When do recursion operators generate new conservation laws?, Phys. Lett. B 277, 137-140 (1992).

[25] O. Mokhov, Symplectic and Poisson Geometry on Loop Spaces of Smooth Manifolds, and Integrable Equations, Reviews in Mathematics and Mathematical Physics, 11, part 2 (2001).

[26] A. Newlander, L. Nirenberg, Complex analytic coordinates in almost complex manifolds, Ann. of Math. 65, 391-404 (1957).

[27] A. Nijenhuis, $X_{n-1}$-forming sets of eigenvectors, Indag. Mathematicae 54, 200-212 (1951).

[28] A. Nijenhuis, Jacobi-type identities for bilinear differential concomitants of certain tensor fields I,II, Indag. Math 17, 390-397, 398-403 (1955).

[29] P. Tempesta, G. Tondo, Haantjes Algebras of Classical Integrable Systems, Preprint arXiv: $1405.5118 \mathrm{v} 3(2019)$.

[30] P. Tempesta, G. Tondo, A New Family of Higher-Order Generalized Haantjes Tensors, Nilpotency and Integrability, Preprint arXiv: 1809.05908v2 (2019).

[31] G. Tondo, Haantjes Algebras of the Lagrange Top, Theor. Math. Phys. 196, 1366-1379 (2018).

[32] G. Tondo, P. Tempesta, Haantjes structures for the Jacobi-Calogero model and the Benenti Systems, SIGMA 12, paper 023, 18 pages (2016). 
Departamento de Física Teórica it, Facultad de Físicas, Universidad Complutense, 28040 - Madrid, Spain and Instituto de Ciencias Matemáticas, C/ Nicolás Cabrera, No 13-15, 28049 MADrid, Spain.

Email address: p.tempesta@fis.ucm.es, piergiulio.tempesta@icmat.es

Dipartimento di Matematica e Geoscienze, Università degli Studi di Trieste, piaz.le Europa 1, I-34127 Trieste, Italy.

Email address: tondo@units.it 